\title{
Consistent 4-form fluxes for maximal supergravity
}

\author{
Hadi Godazgar, ${ }^{a}$ Mahdi Godazgar, ${ }^{a}$ Olaf Krüger $^{b}$ and Hermann Nicolai ${ }^{b}$ \\ ${ }^{a}$ DAMTP, Centre for Mathematical Sciences, University of Cambridge, \\ Wilberforce Road, Cambridge, CB3 0WA, U.K. \\ ${ }^{b}$ Max-Planck-Institut für Gravitationsphysik, Albert-Einstein-Institut, \\ Am Mühlenberg 1, D-14476 Potsdam, Germany \\ E-mail: H.M.Godazgar@damtp.cam.ac.uk, M.M.Godazgar@damtp.cam.ac.uk, \\ Olaf .Krueger@aei.mpg.de, Hermann. Nicolai@aei.mpg.de
}

ABSTRACT: We derive new ansätze for the 4-form field strength of $D=11$ supergravity corresponding to uplifts of four-dimensional maximal gauged supergravity. In particular, the ansätze directly yield the components of the 4-form field strength in terms of the scalars and vectors of the four-dimensional maximal gauged supergravity - in this way they provide an explicit uplift of all four-dimensional consistent truncations of $D=11$ supergravity. The new ansätze provide a substantially simpler method for uplifting $d=4$ flows compared to the previously available method using the 3 -form and 6 -form potential ansätze. The ansatz for the Freund-Rubin term allows us to conjecture a 'master formula' for the latter in terms of the scalar potential of $d=4$ gauged supergravity and its first derivative. We also resolve a long-standing puzzle concerning the antisymmetry of the flux obtained from uplift ansätze.

KeYwords: Supergravity Models, String Duality

ArXIV EPRINT: 1507.07684 


\section{Contents}

1 Introduction 1

2 Preliminaries $\quad 4$

3 Non-linear ansatz for the Freund-Rubin term $\quad 7$

$\begin{array}{ll}3.1 & \text { The 56-bein } \mathcal{V}\end{array}$

3.2 Generalised vielbein postulate 9

$\begin{array}{ll}3.3 \text { The Freund-Rubin term in terms of } d=4 \text { fields } & 11\end{array}$

4 Examples $\quad 13$

4.1 Freund-Rubin term in the $\mathrm{G}_{2}$ invariant sector 13

5 Ansätze for other components of the 4-form field strength 15

6 General form of the Freund-Rubin term $\quad 19$

$\begin{array}{ll}6.1 \text { The conjecture } & 19\end{array}$

6.2 Proof of the conjecture up to quadratic order 20

6.3 Testing the conjecture in the $\mathrm{G}_{2}$ invariant sector 21

$\begin{array}{llr}7 & \text { Outlook } & 23\end{array}$

A Contractions of $\mathrm{G}_{2}$ invariants with Killing forms 24

B Freund-Rubin term in the $\mathrm{SO}(3) \times \mathrm{SO}(3)$ invariant sector 24

C Freund-Rubin term in SU(4)- invariant sector $\quad 25$

\section{Introduction}

Establishing a formal, consistent relation between a higher-dimensional theory and a lower dimensional one is, in general, a challenging problem due to the highly non-linear nature of reductions. Given some (super-)gravity model in $D$ dimensions, consider a ground state solution

$$
\mathcal{M}_{D}=\mathcal{M}_{4} \times \mathcal{M}_{D-4}
$$

corresponding to a compactification from $D$ to four dimensions. The fields of the theory are then expanded linearly around this ground state according to

$$
\Phi(x, y)=\Phi_{0}(x, y)+\sum_{n} \Phi^{(n)}(x) Y^{(n)}(y)
$$


where we collectively denote the value of the fields (metric and form fields) at the ground state by $\Phi_{0}(x, y)$. Here, $x^{\mu}$ and $y^{m}$, respectively, are four-dimensional 'external' and $(D-4)$ dimensional 'internal' coordinates on $\mathcal{M}_{4}$ and $\mathcal{M}_{D-4}$. The $Y^{(n)}(y)$ are the eigenmodes of certain differential operators on the internal space giving rise to an infinite tower of Kaluza-Klein modes. Restricting to the zero-mass eigenmodes gives the low energy physics. The linearised expansion (1.2) is sufficient to determine the mass spectrum of the theory. However, it cannot provide complete information about the interactions of the low energy theory, and must be modified by non-linear terms away from an infinitesimal neighborhood of the ground state. This modification must ensure that any solution of the low energy theory corresponds to a solution of the higher-dimensional theory. This is the problem of Kaluza-Klein consistency: given any solution of the full non-linear field equations in four dimensions one must seek a corresponding expression for $\Phi(x, y)$ that solves the full higher-dimensional field equations also away from $\Phi_{0}(x, y)$, thereby arriving at a consistent embedding of this solution into the higher-dimensional theory.

In fact, there are very few examples where such a program has been successfully completed. Beyond the task of establishing the consistency of the truncation, it is a major challenge to present explicit non-linear ansätze ${ }^{1}$ for uplifting solutions of the lower-dimensional theory to solutions of the higher-dimensional one. Among the known examples the most intricate and technically demanding concerns the maximally supersymmetric $D=11$ supergravity and reductions thereof to maximal gauged supergravity theories in four dimensions, corresponding to the ground state

$$
\mathcal{M}_{11}=\operatorname{AdS}_{4} \times \mathcal{M}_{7}
$$

For this theory the complete non-linear ansätze have recently been identified in refs. [1,2], building on the results of refs. [3-5] and using the formalism developed in ref. [6]. The basic tool that facilitates this result is the reformulation of the $D=11$ supergravity theory [7] such that essential features of maximal gauged supergravity theories, classified by the covariant embedding formalism [8-12], in four dimensions become manifest. At its heart lies the $\mathrm{E}_{7(7)} / \mathrm{SU}(8)$ duality symmetry $[13,14]$, which is obtained in the toroidal reduction from $D=11$ supergravity to four-dimensional ungauged maximal supergravity. An important aspect of the formalism developed in ref. [1] is the role of the 6-form potential, which is dual to the 3 -form potential of $D=11$ supergravity. Ref. [2] (see also refs. $[4,5,15]$ ) derives full, explicit uplift ansätze for $\mathrm{SO}(8)$ gauged maximal supergravity $[16],{ }^{2}$ which is a consistent truncation [3, 21] of $D=11$ supergravity on a seven-sphere [22, 23].

The non-linear ansätze for the internal metric and internal components of the form fields were obtained by an analysis of the supersymmetry variations of $D=11$ supergravity. In particular, the supersymmetry transformation of those components of the fields that we identify with the vectors in a reduction take the same form as the supersymmetry transformation of the vectors in four dimensions, viz. both are given by components of a

\footnotetext{
${ }^{1}$ Here, we use the word "ansatz" in the sense of an approach or prescription rather than a guess.

${ }^{2}$ It is known that the recently discovered family of $\mathrm{SO}(8)$ gauged supergravity theories [17, 18] cannot be obtained from a consistent reduction of $D=11$ supergravity [2, 19, 20] (see also ref. [5]). Therefore, they fall outside the scope of this paper.
} 
56-bein multiplied by a particular combination of fermions. Hence given a linear ansatz for the vectors, one can relate the 56-bein in eleven dimensions to the four-dimensional one. Since these 56 -beine are parametrised by the $d=4$ scalars and the internal components of the $D=11$ fields respectively, one finally obtains a non-linear ansatz that relates the internal components of the $D=11$ fields to the $d=4$ scalars.

By contrast, the approach in this paper is based on an analysis of the generalised vielbein postulates (GVPs). These are analogues of the familiar vielbein postulate in differential geometry for the 56-bein. As in the simpler case of the vielbein postulate, the GVPs express the derivative of the 56-bein in terms of objects that transform as connections with respect to $\mathrm{SU}(8)$ transformations or $\mathrm{E}_{7(7)}$ generalised diffeomorphisms [24]. The GVPs, used in this paper, are found [1] by expressing the 56-bein in a GL(7) decomposition (in terms of the components of the $D=11$ fields) and by packaging its derivative in terms of generalised connections. This alternative method for finding non-linear ansätze (see ref. [3]), centres on the fact that the generalised connections are parametrised by, in particular, components of the 4 -form field strength. Therefore, by projecting onto various components of the GVPs using the 56-bein we are able to extract non-linear ansätze for components of the 4 -form field strength.

One main result of this paper is the embedding formula for the Freund-Rubin parameter $\mathfrak{f}_{F R}(x, y)$ in terms of four-dimensional fields. The latter is generally and independently of the equations of motion defined by [22]

$$
F_{\mu \nu \rho \sigma}(x, y)=i \mathfrak{f}_{F R}(x, y) \stackrel{\circ}{\eta}_{\mu \nu \rho \sigma},
$$

where $\stackrel{\circ}{\eta}_{\mu \nu \rho \sigma}$ is the volume form in four dimensions. The choice of terminology reflects the fact that $\mathfrak{f}_{F R}$ is a constant for Freund-Rubin compactifications characterised by (1.3). On the basis of its observed structure for several examples (worked out in section 4 and appendices $\mathrm{B}$ and $\mathrm{C}$ ) we conjecture the following master formula

$$
\mathfrak{f}_{F R}(x, y)=-\frac{m_{7}}{\sqrt{2} g^{2}}\left(V(x)-\frac{g^{2}}{24}\left(Q^{i j k l}(x) \hat{\Sigma}_{i j k l}(x, y)+\text { h.c. }\right)\right),
$$

where $m_{7}$ is the inverse radius of the round $S^{7}$. Here, $V$ is the full scalar potential of gauged maximal $N=8$ supergravity with gauge coupling constant $g . Q^{i j k l}(x)$ is the first derivative of the potential in an $\mathrm{SU}(8)$ covariant 'frame' on the $\mathrm{E}_{7(7)} / \mathrm{SU}(8)$ coset manifold (see ref. [25] and section 6.1 for details), and $\hat{\Sigma}_{i j k l}$ is the $x$ - and $y$-dependent complex selfdual tensor defined in eq. (6.7) in section 6.1. Stationary points of the potential are therefore characterised by the requirement that $Q^{i j k l}$ be complex anti-selfdual; at such points the $y$-dependence drops out. We perform several very non-trivial checks of the formula (1.5), but leave a general proof for later work.

The master formula (1.5) provides a concrete example of how a higher-dimensional field $\Phi(x, y)$ is consistently deformed away from the ground state solution $\Phi_{0}(x, y)$. At the same time it illustrates very explicitly that the consistency of the truncation can only be achieved on-shell, that is, when the equations of motion are obeyed. Away from the solution of the equations of motion, the Freund-Rubin term exhibits an irremovable and 
manifest $y$-dependence. ${ }^{3}$ The same holds true for other components of the $D=11$ fields, as well as for more complicated solutions of the full $S^{7}$ truncation with $x$-dependence. As we already pointed out in our previous work, this is in marked contrast to the $\operatorname{AdS}_{7} \times S^{4}$ compactification of $D=11$ supergravity $[26,27]$ where there exist consistent non-linear ansätze that also hold off-shell. The reason is that in the latter case the scalar field content is directly obtained without the need to dualise form fields.

Finally, our non-linear ansatz for the internal components $F_{m n p q}$ of the 4-form field strength settles an issue that had been left unresolved in ref. [3], which also tried to exploit the idea of projecting out the 4 -form field strengths from the generalised non-metricity. The construction could not be completed there because only part of the generalised vielbein was known; furthermore, as shown much later in ref. [21], the ansatz as given in ref. [3] yields a tensor that is not totally antisymmetric. We also use the fermion supersymmetry transformations to find an ansatz for the $F_{\mu \nu m n}$ component of the 4-form field strength. With these new ansätze, the uplift of flows ( $x$-dependent solutions) to $D=11$ becomes technically relatively straighforward.

\section{Preliminaries}

A (bosonic) solution of four-dimensional maximal gauged supergravity is specified by the following bosonic field content:

$$
\begin{aligned}
& \text { a vierbein : } \quad e_{\mu}{ }^{\alpha}(x) \text {, } \\
& 28 \text { vector fields : } \quad A_{\mu}^{I J}(x) \text {, } \\
& 70 \text { scalars : } \quad \hat{\mathcal{V}}(x)=\left(\begin{array}{cc}
u_{i j}{ }^{I J}(x) & v_{i j I J}(x) \\
v^{i j I J}(x) & u^{i j}{ }_{I J}(x)
\end{array}\right) \text {, }
\end{aligned}
$$

where the bivector indices $I J$ denote the $\mathbf{2 8}$ of $\mathrm{SL}(8, \mathbb{R})$. The 28 'electric' vector fields $A_{\mu}{ }^{I J}$ should really be thought of as belonging to a $\mathbf{5 6}$ of $\mathrm{E}_{7(7)}$, denoted by $A_{\mu}{ }^{\mathcal{M}}$. In the ungauged theory, the other 28 'magnetic' vectors $A_{\mu I J}$ are obtained by dualising the original 28 vectors $A_{\mu}{ }^{I J}$. The scalars $u_{i j}^{I J}$ and pseudoscalars $v_{i j} I J$ parametrise a coset element $\hat{\mathcal{V}}(x) \in \mathrm{E}_{7(7)} / \mathrm{SU}(8)$.

On the other hand, a solution of $D=11$ supergravity is given by the following bosonic field content:

$$
\begin{array}{ll}
\text { an elfbein : } & E_{M}^{A}(x, y), \\
\text { a 3-form potential : } & A_{M N P}(x, y) \\
\text { (or 4-form field strength : } & \left.F_{M N P Q}(x, y)=24 \partial_{[M} A_{N P Q]}\right),
\end{array}
$$

where $y^{m}$ now are seven-dimensional coordinates.

\footnotetext{
${ }^{3}$ Nevertheless, in the general $S^{7}$ truncation, a residual $y$-dependence of the Freund-Rubin term for nonstationary solutions can be consistent if other components of the 4-form field strength also contribute. Consistency is then achieved because on-shell the $y$-dependence of the latter cancels the residual $y$-dependence of $\mathfrak{f}_{F R}$ in such a way that all these terms combine to sum up to a $y$-independent right-hand side for the $d=4$ Ricci tensor.
} 
An uplift of a four-dimensional solution (2.1) to $D=11$ supergravity is a solution of the $D=11$ equations of motion, specified by (2.2) that is determined purely by the fourdimensional field content (2.1) and the internal geometry of $\mathcal{M}_{7}$ relevant to the reduction; in the case of $\mathrm{SO}(8)$ gauged supergravity this is the seven-sphere $S^{7}$. Decomposing the $D=11$ fields in a $4+7$ split and interpreting them as four-dimensional fields based on their index structure gives:

$$
\begin{array}{lll}
\text { a vierbein : } & e_{\mu}{ }^{\alpha}(x, y), & \\
28 \text { vector fields : } & B_{\mu}{ }^{m}(x, y), & A_{\mu m n}(x, y), \\
70 \text { scalars : } & e_{m}{ }^{a}(x, y), \quad A_{m n p}(x, y), \quad A_{\mu \nu m}(x, y) \quad\left(\text { or } A_{m_{1} \ldots m_{6}}(x, y)\right) .
\end{array}
$$

Modulo a Weyl rescaling the eleven-dimensional "vierbein" (the appropriate $4 \times 4$ submatrix of the elfbein) is simply identified with the four-dimensional one. The 28 vector fields can be augmented by another set of 21 vectors $A_{\mu m_{1} \ldots m_{5}}(x, y)$ originating from the 6 -form dual field $A_{M_{1} \cdots M_{6}}$. The final seven vectors required to form a full $\mathbf{5 6}$ of $\mathrm{E}_{7(7)}$ correspond to the seven 'dual graviphotons' that have no satisfactory interpretation within $D=11$ supergravity. Nevertheless, for convenience, we can add seven extra auxiliary vectors (see e.g. ref. [1] and references therein). In this way we collectively define a set of vectors $B_{\mu}{ }^{\mathcal{M}}$, where indices $\mathcal{M}, \mathcal{N}, \ldots$ label the $\mathbf{5 6}$ representation of $\mathrm{E}_{7(7)}$. These vectors are related to the analogous set $A_{\mu}{ }^{\mathcal{M}}$ in four dimensions by

$$
B_{\mu}{ }^{\mathcal{M}}(x, y)=\mathcal{R}^{\mathcal{M}}{ }_{\mathcal{N}}(y) A_{\mu}{ }^{\mathcal{N}}(x) \equiv \mathcal{R}^{\mathcal{M}}{ }_{I J}(y) A_{\mu}{ }^{I J}(x)+\mathcal{R}^{\mathcal{M} I J}(y) A_{\mu I J}(x) .
$$

Here, $A_{\mu}{ }^{I J}$ and $A_{\mu I J}$, respectively, are the 28 electric vectors and the 28 magnetic vectors of $N=8$ supergravity. In the case of the $S^{7}$ reduction, $\mathcal{R}^{\mathcal{M}}{ }_{\mathcal{N}}$ is constructed from the Killing spinors $\eta^{I}$ on $S^{7}$ and the 6 -form volume potential on the round $S^{7}, \stackrel{\circ}{\zeta}_{m_{1} \ldots m_{6}}$; the explicit expressions are given in ref. [2]. Similarly, the eleven-dimensional "scalars," which collectively define an $\mathrm{E}_{7(7)} / \mathrm{SU}(8)$ coset element $\mathcal{V}^{\mathcal{M}}{ }_{A B}[1]$ are related to the four-dimensional scalars via

$$
\mathcal{V}^{\mathcal{M}}{ }_{A B}(x, y)=\mathcal{R}^{\mathcal{M}}{ }_{\mathcal{N}}(y) \eta_{A}^{i}(y) \eta_{B}^{j}(y) \hat{\mathcal{V}}^{\mathcal{N}}{ }_{i j}(x) .
$$

Here, $\eta_{A}^{i}$ denote the eight Killing spinors defined on the internal geometry and $\mathcal{R}^{\mathcal{M}}{ }_{\mathcal{N}}$ is the same matrix as in eq. (2.4).

In the case of the $S^{7}$ reduction and the associated $\mathrm{SO}(8)$ gauged supergravity, the above expressions translate to an uplift ansatz for the internal metric $g_{m n}$, [4], the internal 3 -form potential $A_{m n p}[2,5,15]$ and the internal 6-form potential $A_{m_{1} \ldots m_{6}}[2]$. Furthermore, dualisation of the 6 -form potential gives components of the 3 -form potential. All these fields obtained in this way represent a full constructive solution of the $D=11$ equations of motion. The two-form fields $A_{\mu \nu m}$ can be obtained by integration from the other ansätze. It is in principle also possible to deduce a non-linear ansatz directly for $A_{\mu \nu m}$ by also comparing the four and eleven-dimensional supersymmetry transformations. Except that in this case the supersymmetry transformation of $A_{\mu \nu m}$ will correspond in four dimensions to the supersymmetry transformation of the $\mathbf{1 3 3}$ two-form fields, $A_{\mu \nu \boldsymbol{\alpha}}$, in the tensor hierarchy (see ref. [12]). 
It must be emphasised that the uplift ansätze have been derived from the $D=11$ theory, with the supersymmetry transformations playing a significant role in the derivation. As such they are robust and need no further substantiation. However, given the non-trivial nature of the reduction on the one hand and the remarkably simple form of the ansätze on the other, they have been explicitly verified for a number of stationary points of the fourdimensional scalar potential including the $\mathrm{SO}(7)^{ \pm}, \mathrm{G}_{2}$ and $\mathrm{SU}(4)^{-}$invariant solutions $[2,4$, 15, 21]. Furthermore, the metric ansatz has been used extensively in the literature, in particular in applications to holography (see for example [28, 29]). The full uplift ansätze have allowed for a study of more complicated upliftings; including an uplift of the $\mathrm{SO}(3) \times \mathrm{SO}(3)$ invariant solution [30] and for the first time a full uplift of a flow to eleven dimensions [31].

In this paper, we explore the possibility of expressing some of the uplift ansätze in even simpler terms, with particular focus on the Freund-Rubin term (1.4) that plays a central role in compactifications of $D=11$ supergravity. To illustrate the simplicity of our final formula (1.5) recall the duality relation in eleven dimensions between the 4 -form field strength and its 7 -form dual, which implies that the Freund-Rubin term can also be expressed (in form language) as

$$
\mathfrak{f}_{F R}=\star_{(7)}\left(\mathrm{d}_{(7)} A_{6}-A_{3} \wedge F_{4}\right),
$$

where all fields above take components along the internal directions. Hence, a direct derivation of the Freund-Rubin term from the uplift ansätze of ref. [2] would require the associated expressions for $A_{m n p}$ and $A_{m_{1} \ldots m_{6}}$. Although eq. (2.6) and the uplift ansätze for $A_{m n p}$ and $A_{m_{1} \ldots m_{6}}$ are relatively simple, ${ }^{4}$ in practice the calculations become rather unwieldy for more non-trivial solutions of the four-dimensional theory, at least analytically. More precisely, the large number of operations required (such as inverting the metric to find $A_{m n p}$ and $A_{m_{1} \ldots m_{6}}$, taking exterior derivatives and dualising a 7 -form) to find what is ultimately a scalar makes it a rather inconvenient calculation.

Observing that the Freund-Rubin term, as well as other components of the 4-form field strength, also appear in the generalised vielbein postulates (GVPs) $[1,6]$, and more specifically, in the generalised $\mathrm{SU}(8)$ connection coefficients $\mathcal{Q}_{m}{ }^{A} B$ and the generalised non-metricity $\mathcal{P}_{m A B C D}$, we obtain (in our view the rather elegant) formula (1.5) for $\mathfrak{f}_{F R}$ that is sextic in the matrix elements of $\hat{\mathcal{V}}$, see eq. (3.40), by a particular projection of the internal GVP using components of the 56-bein.

Another projection of the internal GVP gives an ansatz for the internal components of the field strength. When projecting out $F_{m n p q}$ from the generalised non-metricity $\mathcal{P}_{m} A B C D$, components of the generalised Christoffel connection $\boldsymbol{\Gamma}_{m \mathcal{N}}^{\mathcal{P}}$ contribute, see eq. (5.2). In fact these terms, which correspond to ambiguities in the language of ref. [21], remove all terms in $F_{m n p q}$ that are not fully antisymmetric so that $F_{m n p q}=F_{[m n p q]}$, as required by its compatibility with (2.2). Note that, when projecting out the Freund-Rubin term $\mathfrak{f}_{F R}$ from

\footnotetext{
${ }^{4}$ In fact, the ansatz for $A_{m_{1} \ldots m_{6}}$ given in ref. [2] can be greatly simplified:

$$
A_{m_{1} \ldots m_{6}}=\frac{1}{m_{7}} \frac{\sqrt{2}}{16 \cdot 5 !} \stackrel{\circ}{g}^{p q}{\stackrel{\circ}{\eta} m_{1} \ldots m_{6} p}_{D_{q}}(\log \Delta)-3 \sqrt{2} \stackrel{\circ}{\zeta}_{m_{1} \ldots m_{6}}
$$
}


the generalised vielbein postulate, components of the generalised Christoffel connection drop out. In this way we are finally able to resolve an issue that was left unfinished in ref. [3]: it is also observed there that one can project out the 4-form field strength. However, the resulting $\mathrm{SU}(8)$ invariant expression, apart from the ambiguities pointed out in [21], turns out to be unmanageably complicated due to the fact that only part of the generalised vielbein was known. Nevertheless we can now confirm that this strategy is correct, and does yield non-linear ansätze for the field strengths of the form fields. In particular, these new ansätze can be more suitable than using the ones for the form fields themselves.

Furthermore, in section 5, we use the external GVP and the fermion supersymmetry transformations to find ansätze for the remaining components of the field strength. In particular, we find new direct and simple ansätze for the $F_{\mu \nu \rho m}$ and $F_{\mu \nu m n}$ components, eqs. (5.15) and (5.22), respectively. We verify the ansatz for $F_{\mu \nu \rho m}$ for the $\mathrm{SO}(7)^{+}$sector.

\section{Non-linear ansatz for the Freund-Rubin term}

\subsection{The 56-bein $\mathcal{V}$}

The internal components of the $D=11$ fields are packaged into a single coset element of $\mathrm{E}_{7(7)} / \mathrm{SU}(8)$, a 56 -bein $\left(\mathcal{V}_{\mathcal{M}}{ }^{A B}, \mathcal{V}_{\mathcal{M} A B}\right)$. Here, the $\mathrm{E}_{7(7)}$ index $\mathcal{M}$ decomposes under GL(7) as

$$
\mathcal{V}_{\mathcal{M}}=\left(\mathcal{V}_{m 8}, \mathcal{V}_{m n}, \mathcal{V}^{m n}, \mathcal{V}^{m 8}\right)
$$

with GL(7) indices $m, n, \cdots=1, \ldots, 7$. The components of $\mathcal{V}$ in terms of the $D=11$ fields are [1],

$$
\begin{aligned}
& \mathcal{V}^{m 8}{ }_{A B}=-\frac{\sqrt{2}}{8} \Delta^{-1 / 2} \Gamma_{A B}^{m}, \\
& \mathcal{V}_{m n A B}=-\frac{\sqrt{2}}{8} \Delta^{-1 / 2}\left(\Gamma_{m n A B}+6 \sqrt{2} A_{m n p} \Gamma_{A B}^{p}\right), \\
& \mathcal{V}_{A B}^{m n}=-\frac{\sqrt{2}}{8} \cdot \frac{1}{5 !} \grave{\eta}^{m n p_{1} \cdots p_{5}} \Delta^{-1 / 2}\left[\Gamma_{p_{1} \cdots p_{5} A B}+60 \sqrt{2} A_{p_{1} p_{2} p_{3}} \Gamma_{p_{4} p_{5}} A B\right. \\
& \left.-6 ! \sqrt{2}\left(A_{q p_{1} \cdots p_{5}}-\frac{\sqrt{2}}{4} A_{q p_{1} p_{2}} A_{p_{3} p_{4} p_{5}}\right) \Gamma_{A B}^{q}\right], \\
& \mathcal{V}_{m 8 A B}=-\frac{\sqrt{2}}{8} \cdot \frac{1}{7 !} \grave{\eta}^{p_{1} \cdots p_{7}} \Delta^{-1 / 2}\left[\left(\Gamma_{p_{1} \cdots p_{7}} \Gamma_{m}\right)_{A B}+126 \sqrt{2} A_{m p_{1} p_{2}} \Gamma_{p_{3} \cdots p_{7}} A B\right. \\
& +3 \sqrt{2} \cdot 7 !\left(A_{m p_{1} \cdots p_{5}}+\frac{\sqrt{2}}{4} A_{m p_{1} p_{2}} A_{p_{3} p_{4} p_{5}}\right) \Gamma_{p_{6} p_{7} A B} \\
& \left.+\frac{9 !}{2}\left(A_{m p_{1} \cdots p_{5}}+\frac{\sqrt{2}}{12} A_{m p_{1} p_{2}} A_{p_{3} p_{4} p_{5}}\right) A_{p_{6} p_{7} q} \Gamma_{A B}^{q}\right] .
\end{aligned}
$$

Here, $\Gamma^{a_{1} \cdots a_{n}}=\Gamma^{\left[a_{1}\right.} \ldots \Gamma^{\left.a_{n}\right]}$ are seven-dimensional $8 \times 8 \Gamma$-matrices and $\Gamma^{m_{1} \cdots m_{n}}$ are their curved versions, e.g. $\Gamma^{m}=e^{m}{ }_{a} \Gamma^{a}$. $A_{p q r}$ and $A_{m_{1} \cdots m_{6}}$ are 3 -form and 6-form fields, respectively. The $\mathcal{V}$ given above is an $\mathrm{E}_{7(7)}$ matrix because it corresponds to the exponentiation of $\mathrm{E}_{7(7)}$ Lie algebra elements [32]. 
The index $\mathcal{M}$ that denotes the $\mathbf{5 6}$ of $\mathrm{E}_{7(7)}$ is raised and lowered with the symplectic metric $\Omega^{\mathcal{M N}}$ and its inverse, namely

$$
\mathcal{V}^{\mathcal{M}}=\Omega^{\mathcal{M N}} \mathcal{V}_{\mathcal{N}}
$$

The non-vanishing components of $\Omega^{\mathcal{M N}}$ are

$$
\Omega_{p q}^{m n}=-\Omega_{p q}^{m n}=\delta_{p q}^{m n}, \quad \Omega_{p 8}^{m 8}=-\Omega_{p 8}^{m 8}=\delta_{p 8}^{m 8}=\frac{1}{2} \delta_{p}^{m}
$$

and its inverse is defined by

$$
\Omega^{\mathcal{M P}} \Omega_{\mathcal{N P}}=\delta_{\mathcal{N}}^{\mathcal{M}}
$$

$\left(\mathcal{V}_{\mathcal{M}}^{A B} \mathcal{V}_{\mathcal{M} A B}\right)$ is an $\operatorname{Sp}(56, \mathbb{R})$ matrix and hence

$$
\mathcal{V}^{\mathcal{M} A B} \mathcal{V}_{\mathcal{M} C D}=i \delta_{C D}^{A B}, \quad \mathcal{V}^{\mathcal{M} A B} \mathcal{V}_{\mathcal{M}}{ }^{C D}=0
$$

'Curved' $\mathrm{SU}(8)$ indices $A, B, \ldots$ are raised and lowered by complex conjugation,

$$
\mathcal{V}_{\mathcal{M}}{ }^{A B}=\left(\mathcal{V}_{\mathcal{M} A B}\right)^{*}, \quad \mathcal{V}^{\mathcal{M} A B}=\left(\mathcal{V}_{A B}^{\mathcal{M}}\right)^{*},
$$

while the position of the $\mathrm{E}_{7(7)}$ index on $\mathcal{V}$ is not affected.

The $D=1156$-bein is related via the linear ansatz (2.5) [1] to the $\mathrm{E}_{7(7)}$ matrix that encodes the scalars of $N=8$ supergravity

$$
\hat{\mathcal{V}}=\left(\begin{array}{cc}
u_{i j}^{I J} & v_{i j I J} \\
v^{i j I J} & u^{i j} I J
\end{array}\right) .
$$

The 70 scalars and pseudoscalars parametrise $u_{i j}{ }^{I J}(x)$ and $v_{i j} I J(x)$. In the form above, the 56-bein is given in an $\mathrm{SU}(8)$ basis. However, it turns out to be more convenient to have the 56 -bein such that its $\mathrm{E}_{7(7)}$ index is decomposed in an $\mathrm{SL}(8)$ basis: $^{5}$

$$
\hat{\mathcal{V}}_{\mathcal{M}}{ }^{i j}=\frac{1}{\sqrt{2}}\left(\begin{array}{c}
u^{i j}{ }_{I J}-v^{i j I J} \\
-i\left(u^{i j}{ }_{I J}+v^{i j I J}\right)
\end{array}\right), \quad \hat{\mathcal{V}}_{\mathcal{M} i j} \equiv\left(\hat{\mathcal{V}}_{\mathcal{M}}{ }^{i j}\right)^{*}=\frac{1}{\sqrt{2}}\left(\begin{array}{c}
u_{i j}{ }^{I J}-v_{i j I J} \\
i\left(u_{i j}{ }^{I J}+v_{i j I J}\right)
\end{array}\right) \text {. }
$$

In relating the $d=456$-bein to the eleven-dimensional one given above, one must in principle take into account a compensating $\mathrm{SU}(8)$ rotation depending on all eleven coordinates, as explained in ref. [6]. However, in the remainder we will deal only with quantities where the $\mathrm{SU}(8)$ indices are fully contracted, and this $\mathrm{SU}(8)$ rotation drops out. Keeping this in mind, the explicit dependence of the components on the $d=4$ fields is [2]

$$
\begin{aligned}
& \mathcal{V}^{m 8}{ }_{i j}(x, y)=\frac{\sqrt{2} i}{8} K^{m I J}(y)\left(u_{i j}^{I J}+v_{i j I J}\right)(x), \\
& \mathcal{V}_{m n i j}(x, y)=-\frac{\sqrt{2}}{8} K_{m n}{ }^{I J}(y)\left(u_{i j}{ }^{I J}-v_{i j I J}\right)(x), \\
& \mathcal{V}^{m n}{ }_{i j}(x, y)=\frac{\sqrt{2} i}{8 \cdot 5 !} i^{m n p_{1} \cdots p_{5}}\left(K_{p_{1} \cdots p_{5}}^{I J}-6 \cdot 6 !{\stackrel{\circ}{\zeta_{1} \cdots p_{5} q}} K^{q I J}\right)(y)\left(u_{i j}{ }^{I J}+v_{i j} I J\right)(x),
\end{aligned}
$$

\footnotetext{
${ }^{5}$ See, for example, ref. [33] for more explanation.
} 


$$
\mathcal{V}_{m 8 i j}(x, y)=\frac{\sqrt{2}}{8}\left(K_{m}{ }^{I J}+6 \dot{\eta}^{p_{1} \cdots p_{7}}{\stackrel{\circ}{\zeta_{1} \cdots p_{6}}}_{K_{p_{7} m}{ }^{I J}}\right)(y)\left(u_{i j}{ }^{I J}-v_{i j I J}\right)(x),
$$

where $K_{m}^{I J}(y)$ are the Killing vectors on the round seven-sphere,

$$
\begin{aligned}
K_{m}{ }^{I J} & =i \bar{\eta}^{I} \Gamma_{m} \eta^{J}, \quad K_{n}{ }^{I J}=-\frac{1}{m_{7}} \stackrel{\circ}{D}_{m} K_{n}^{I J}=\bar{\eta}^{I} \Gamma_{m n} \eta^{J} \\
K_{m_{1} \ldots m_{5}}{ }^{I J} & =i \bar{\eta}^{I} \Gamma_{m_{1} \cdots m_{5}} \eta^{J}=-\frac{1}{2} \grave{\eta}_{m_{1} \cdots m_{7}} K^{m_{6} m_{7} I J} .
\end{aligned}
$$

The derivative operator $\stackrel{\circ}{D}_{m}$ is the covariant derivative with respect to the Christoffel connection on the round sphere and $\eta^{I}$ are the eight Killing spinors on $S^{7}$. Additionally,

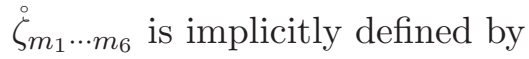

$$
7 ! \stackrel{\circ}{D}_{\left[m_{1}\right.} \stackrel{\circ}{\zeta}_{\left.m_{2} \cdots m_{7}\right]}=m_{7} \stackrel{\circ}{\eta}_{m_{1} \cdots m_{7}}
$$

Furthermore the normalisations in (3.11)-(3.14) have been chosen so that this vielbein is indeed normalised according to eq. (3.7). These expressions are sufficient to derive all non-linear ansätze.

\subsection{Generalised vielbein postulate}

The generalised vielbein postulates are differential constraints on the 56-bein in terms of generalised connections including an $\mathrm{SU}(8)$ connection, a generalised $\mathrm{E}_{7(7)}$ connection and a generalised non-metricity. Using the GL(7) decomposition of the 56-bein, (3.5), its derivative can be grouped into objects that satisfy the correct transformation properties, namely the generalised connections in refs. $[1,6]$. The crucial feature of the generalised connections that we utilise in order to derive our ansätze is that they are parametrised by components of the 4 -form field strength. This is a somewhat different approach to the deductive approach of ref. [24]. There, the generalised connections are found by requiring a torsion-free compatible connection (in contrast to usual differential geometry, this does not uniquely specify the connections [24]). The generalised connections in ref. [24] are nevertheless related [34] to the generalised connections in ref. [1]; as are the connections in exceptional field theory [34], where the emphasis is on connections that are expressed in terms of the 56-bein of exceptional field theory [35].

A distinctive feature of the generalised connections that we use is that they are valued along the first seven directions in a GL(7) decomposition, as is clear from eq. (3.18). Note that this is not a consequence of the derivative index running over seven directions, but rather a consequence of working with a generalised non-metricity rather than torsion-free compatible connections [34], which are valued in the $\mathbf{5 6}$ even when the base space is not extended as in ref. [24]. However, for us it is precisely the SU(8) covariant generalised non-metricity that yields the new non-linear ansätze.

The 56-bein $\mathcal{V}_{\mathcal{M}}$ satisfies, in particular, the internal GVP $[1,36]$

$$
\partial_{m} \mathcal{V}_{\mathcal{N} A B}-\boldsymbol{\Gamma}_{m \mathcal{N}} \mathcal{P}^{\mathcal{P} A B}+\mathcal{Q}_{m[A}^{C} \mathcal{V}_{\mathcal{N} B] C}=\mathcal{P}_{m A B C D} \mathcal{V}_{\mathcal{N}}{ }^{C D}
$$


where $\mathcal{Q}_{m B}^{A}$ is the generalised $\mathrm{SU}(8)$ connection. The $\mathrm{SU}(8)$ tensor $\mathcal{P}_{m A B C D}$ is the 'generalised non-metricity', which 'measures' the failure of the metric

$$
\mathcal{G}_{\mathcal{M N}} \equiv \mathcal{V}_{\mathcal{M}}{ }^{A B} \mathcal{V}_{\mathcal{N} A B}+\mathcal{V}_{\mathcal{N}}{ }^{A B} \mathcal{V}_{\mathcal{M} A B}
$$

to be covariantly constant under the generalised covariant derivative. ${ }^{6} \boldsymbol{\Gamma}_{m \mathcal{N}}{ }^{\mathcal{P}}$ is the $\mathrm{E}_{7(7)}$ generalised Christoffel connection with components

$$
\begin{aligned}
& \left(\boldsymbol{\Gamma}_{m}\right)_{p 8}^{q 8}=-\left(\boldsymbol{\Gamma}_{m}\right)^{q 8}{ }_{p 8}=\frac{1}{2} \Gamma_{m p}^{q}+\frac{1}{4} \Gamma_{m n}^{n} \delta_{p}^{q}, \quad\left(\boldsymbol{\Gamma}_{m}\right)_{p q}^{r s}=-\left(\boldsymbol{\Gamma}_{m}\right)^{r s}{ }_{p q}=2 \Gamma_{m[p}^{[r} \delta_{q]}^{s]}-\frac{1}{2} \Gamma_{m n}^{n} \delta_{p q}^{r s}, \\
& \left(\boldsymbol{\Gamma}_{m}\right)_{p 8}{ }^{r s}=-\left(\boldsymbol{\Gamma}_{m}\right)^{r s}{ }_{p 8}=3 \sqrt{2} \eta^{r s t_{1} \cdots t_{5}} \Xi_{m \mid p t_{1} \cdots t_{5}}, \\
& \left(\boldsymbol{\Gamma}_{m}\right)_{p q r 8}=\left(\boldsymbol{\Gamma}_{m}\right)_{r 8 p q}=3 \sqrt{2} \Xi_{m \mid p q r}, \quad\left(\boldsymbol{\Gamma}_{m}\right)^{p q r s}=\frac{1}{\sqrt{2}} \eta^{p q r s t_{1} t_{2} t_{3}} \Xi_{m \mid t_{1} t_{2} t_{3}} .
\end{aligned}
$$

Here,

$$
\Gamma_{m n}^{p}(x, y) \equiv \frac{1}{2} g^{p q}\left(\partial_{m} g_{n q}+\partial_{n} g_{m q}-\partial_{q} g_{m n}\right)
$$

denotes the usual Christoffel connection defined with respect to the metric $g_{m n}(x, y)$. The quantities $\Xi_{m \mid p q r}(x, y)$ and $\Xi_{m \mid p_{1} \cdots p_{6}}(x, y)$ are [36]

$$
\begin{aligned}
\Xi_{m \mid p q r}=D_{m} A_{p q r}- & \frac{1}{4 !} \\
\Xi_{m \mid p_{1} \cdots p_{6}}=D_{m} A_{p_{1} \cdots p_{6}} & +\frac{\sqrt{2}}{48} F_{m\left[p_{1} p_{2} p_{3}\right.} A_{\left.p_{4} p_{5} p_{6}\right]} \\
& -\frac{\sqrt{2}}{2}\left(D_{m} A_{\left[p_{1} p_{2} p_{3}\right.}-\frac{1}{4 !} F_{m\left[p_{1} p_{2} p_{3}\right.}\right) A_{\left.p_{4} p_{5} p_{6}\right]}-\frac{1}{7 !} F_{m p_{1} \cdots p_{6}}
\end{aligned}
$$

where $D_{m}$ denotes the covariant derivative with respect to the Christoffel connection $\Gamma_{m n}^{p}$. From the definitions above it is clear that

$$
\Xi_{[m \mid n p q]}=\Xi_{\left[m \mid p_{1} \cdots p_{6}\right]}=0 .
$$

We also note that under generalised diffeomorphisms (including the two- and five-form gauge transformations) all connection coefficients transform with second derivatives, just like the standard Christoffel connection.

In a non-trivial background (such as the compactification on $S^{7}$ ), all $\mathrm{E}_{7(7)}$ Christoffel connections decompose into a background connection $\stackrel{\circ}{\boldsymbol{\Gamma}}_{m \mathcal{N}}^{\mathcal{P}}$ and a variation $\hat{\boldsymbol{\Gamma}}_{m \mathcal{N}}^{\mathcal{P}}$,

$$
\boldsymbol{\Gamma}_{m \mathcal{N}}^{\mathcal{P}}=\stackrel{\circ}{\Gamma}_{m \mathcal{N}}^{\mathcal{P}}+\hat{\boldsymbol{\Gamma}}_{m \mathcal{N}^{\mathcal{P}}}
$$

For the $S^{7}$ compactification we will see, eqs. (3.30)-(3.32), that the background connection is not only given by the standard covariantisation with respect to $\stackrel{\circ}{\Gamma}_{m n}^{p}$, but that it also requires a non-vanishing component $\stackrel{\circ}{\Xi}_{m \mid p_{1} \cdots p_{6}}$.

\footnotetext{
${ }^{6}$ As explained in ref. [34] the non-metricity can be absorbed into the connections, at the price of introducing components $\mathcal{Q}_{\mathcal{M B}}^{A}$ and $\Gamma_{\mathcal{M N}}^{\mathcal{P}}$ along directions $\mathcal{M} \neq m$.
} 
The generalised spin connection $\mathcal{Q}_{m B}^{A}$ and non-metricity $\mathcal{P}_{m} A B C D$ are expressed in terms of the $D=11$ fields as follows [6]:

$$
\begin{aligned}
\mathcal{Q}_{m B}^{A} & =-\frac{1}{2} \omega_{m a b} \Gamma_{A B}^{a b}+\frac{\sqrt{2}}{14} i \Delta^{2} \mathfrak{f}_{F R} \Gamma_{m A B}-\frac{\sqrt{2}}{48} F_{m n p q} \Gamma_{A B}^{n p q}, \\
\mathcal{P}_{m A B C D} & =\frac{\sqrt{2}}{56} i \Delta^{2} \mathfrak{f}_{F R} \Gamma_{m n[A B} \Gamma_{C D]}^{n}+\frac{\sqrt{2}}{32} F_{m n p q} \Gamma_{[A B}^{n} \Gamma_{C D]}^{p q},
\end{aligned}
$$

where $\omega_{m a b}$ is the $\mathrm{SO}(7)$ spin-connection. The internal GVP, (3.18), provides a non-linear ansatz for $\mathfrak{f}_{F R}$, given that $\mathcal{P}_{m}$ depends on $\mathfrak{f}_{F R}$. From eq. (3.18), we find

$$
\mathcal{P}_{m A B C D}=-i \mathcal{V}^{\mathcal{N}}{ }_{C D} \mathbf{D}_{m} \mathcal{V}_{\mathcal{N} A B} \equiv-i \mathcal{V}^{\mathcal{N}}{ }_{C D} \partial_{m} \mathcal{V}_{\mathcal{N} A B}+i \boldsymbol{\Gamma}_{m \mathcal{N}^{\mathcal{P}}} \mathcal{V}^{\mathcal{N}}{ }_{C D} \mathcal{V}_{\mathcal{P} A B}
$$

and project out the Freund-Rubin term using the $D=11$ vielbein components,

$$
\mathfrak{f}_{F R}=-\frac{8 \sqrt{2} i}{3} \mathcal{V}^{m 8 E F} \mathcal{V}^{p 8}{ }_{E F} \mathcal{V}_{p q}{ }^{A B} \mathcal{V}^{q 8 C D} \mathcal{P}_{m A B C D}
$$

Note that in eq. (3.25), we defined the full covariant derivative $\mathbf{D}_{m}$ with respect to the full $\mathrm{E}_{7(7)}$ Christoffel connection. We denote the covariant derivative associated with the full background connection $\stackrel{\circ}{\Gamma}_{m \mathcal{N}}^{\mathcal{P}}, \stackrel{\circ}{\mathbf{D}}_{m}$.

Substituting the expression for $\mathcal{P}_{m}$ from eq. (3.25), this projection has the following convenient property: as a result of contracting out all $\mathrm{SU}(8)$ indices all the generalised connection components (3.19) drop out in $\mathfrak{f}_{F R}$. For this reason we can use any connection; we choose to work with the background connection for convenience. Note that this is not true for other projections, in particular the 4-form field strength $F_{m n p q}$. In section 7 , we give a new ansatz for $F_{m n p q}$ that takes these "ambiguities" into account. Thus,

$$
\mathfrak{f}_{F R}=-\frac{8 \sqrt{2}}{3} \mathcal{V}^{m 8 E F} \mathcal{V}^{p 8}{ }_{E F} \mathcal{V}_{p q}{ }^{A B} \mathcal{V}^{q 8 C D} \mathcal{V}^{\mathcal{N}}{ }_{C D} \stackrel{\circ}{\mathbf{D}}_{m} \mathcal{V}_{\mathcal{N} A B}
$$

\subsection{The Freund-Rubin term in terms of $d=4$ fields}

We convert curved $\mathrm{SU}(8)$ indices $A, B, \ldots$ into flat $\mathrm{SU}(8)$ indices $i, j, \ldots$ (cf. eq. (2.5)) by means of the orthonormal Killing spinors on the round sphere $\eta_{A}^{i}$,

$$
\begin{aligned}
\mathcal{P}_{m i j k l} & =-i \mathcal{V}^{\mathcal{N}}{ }_{k l}{\stackrel{\circ}{\mathbf{D}_{m}}} \mathcal{V}_{\mathcal{N} i j}+i \hat{\boldsymbol{\Gamma}}_{m \mathcal{N}^{\mathcal{P}}} \mathcal{V}^{\mathcal{N}}{ }_{k l} \mathcal{V}_{\mathcal{P}}{ }_{i j} \\
\mathfrak{f}_{F R} & =-\frac{8 \sqrt{2}}{3} \mathcal{V}^{m 8 r s} \mathcal{V}^{p 8}{ }_{r s} \mathcal{V}_{p q}{ }^{i j} \mathcal{V}^{q 8}{ }^{k l} \mathcal{V}^{\mathcal{N}}{ }_{k l}{\stackrel{\circ}{\mathbf{D}_{m}}}_{\mathcal{V}_{\mathcal{N}} i j}
\end{aligned}
$$

Here, we used the split (3.22) for the $S^{7}$ background, with the only non-vanishing Christoffel connection components

$$
\begin{aligned}
& \left(\stackrel{\circ}{\Gamma}_{m}\right)_{p 8}^{q 8}=-\left(\stackrel{\circ}{\boldsymbol{\Gamma}}_{m}\right)^{q 8}{ }_{p 8}=\frac{1}{2} \stackrel{\circ}{\Gamma}_{m p}^{q}+\frac{1}{4} \stackrel{\circ}{\Gamma}_{m n}^{n} \delta_{p}^{q}, \\
& \left(\stackrel{\circ}{\boldsymbol{\Gamma}}_{m}\right)_{p q}{ }^{r s}=-\left(\stackrel{\circ}{\boldsymbol{\Gamma}}_{m}\right)^{r s}{ }_{p q}=2 \stackrel{\circ}{\Gamma}_{m[p}^{[r} \delta_{q]}^{s]}-\frac{1}{2} \stackrel{\circ}{\Gamma}_{m n}^{n} \delta_{p q}^{r s}
\end{aligned}
$$

and

$$
\left(\stackrel{\circ}{\Gamma}_{m}\right)_{p 8}^{r s}=-\left(\stackrel{\circ}{\Gamma}_{m}\right)^{r s}{ }_{p 8}=3 \sqrt{2} \eta^{r s t_{1} \cdots t_{5}} \stackrel{\circ}{\Xi}_{m \mid p t_{1} \cdots t_{5}},
$$


with

$$
\stackrel{\circ}{\Xi}_{m \mid n_{1} \cdots n_{6}} \equiv \stackrel{\circ}{D}_{m} \stackrel{\circ}{A}_{n_{1} \cdots n_{6}}-\stackrel{\circ}{D}_{[m} \stackrel{\circ}{A}_{\left.n_{1} \cdots n_{6}\right]}=3 \sqrt{2}\left(\stackrel{\circ}{D}_{[m} \stackrel{\circ}{\zeta}_{\left.n_{1} \cdots n_{6}\right]}-\stackrel{\circ}{D}_{m} \stackrel{\circ}{\zeta}_{n_{1} \cdots n_{6}}\right) .
$$

Thus, the evaluation of the Freund-Rubin term requires an evaluation of the MaurerCartan form of the 56-bein. This can simply be calculated using eqs. (3.11)-(3.14),

$$
\begin{aligned}
\mathcal{V}^{\mathcal{N}}{ }_{k l} \stackrel{\circ}{D}_{m} \mathcal{V}_{\mathcal{N} i j}= & \frac{3}{28} i m_{7} K_{m n}{ }^{[I J} K^{n K L]}\left(u_{i j}{ }^{I J} u_{k l}{ }^{K L}-v_{i j I J} v_{k l K L}\right) \\
& +\frac{4}{7} i m_{7} K_{m}{ }^{I J}\left(v_{i j M J} u_{k l}{ }^{M I}-u_{i j}{ }^{M J} v_{k l M I}\right) \\
& -12 \stackrel{\circ}{\eta}^{n_{1} \ldots n_{7}}\left(\stackrel{\circ}{D}_{m} \stackrel{\circ}{\zeta}_{n_{1} \ldots n_{6}}-\stackrel{\circ}{D}_{[m} \stackrel{\circ}{\zeta}_{\left.n_{1} \ldots n_{6}\right]}\right)\left(\mathcal{V}^{p 8}{ }_{i j} \mathcal{V}_{n_{7} p k l}+\mathcal{V}^{p 8}{ }_{k l} \mathcal{V}_{n_{7} p i j}\right)
\end{aligned}
$$

where $\stackrel{\circ}{D}_{m}$ is the usual $S^{7}$ covariant derivative. The last term on the right-hand side of the above expression exactly cancels the contribution of the generalised connection term coming from $\stackrel{\circ}{\Xi}_{m \mid p_{1} \cdots p_{6}}$ evaluated in $\mathcal{V}^{\mathcal{N}}{ }_{k l} \stackrel{\circ}{D}_{m} \mathcal{V}_{\mathcal{N} i j}$. Namely, at the background value of the fields, where $\hat{\boldsymbol{\Gamma}}_{m \mathcal{N}}^{\mathcal{P}}=0, \mathcal{P}_{m i j k l}$ given by eq. (3.28) is equal to the first two terms in eq. (3.34), reproducing the solution given in equation (3.19) of ref. [3]. ${ }^{7}$ Otherwise, away from the $\mathrm{SO}(8)$ invariant vacuum, the solution is modified by the generalised connection terms $\hat{\boldsymbol{\Gamma}}_{m \mathcal{N}}^{\mathcal{P}}$. These are the "ambiguities" that leave the supersymmetry transformations unchanged [21]. Therefore, the solution proposed in ref. [3] is consistent with the supersymmetry transformations but does not reproduce the field strength components $F_{m n p q}$. In generalised geometry, this is manifested in the lack of a unique torsion-free, metriccompatible generalised connection [24]; see also ref. [34] where this relation was explored.

In fact, equation (3.34) points to the necessity of using a background connection that accounts for the fact that the Freund-Rubin parameter is non-zero at the background. This background connection includes generalised connection components such that

$$
\begin{aligned}
\mathcal{V}^{\mathcal{N}}{ }_{k l}{\stackrel{\circ}{\mathbf{D}_{m}}}_{\mathcal{V}_{\mathcal{N}} i j}= & \frac{3}{28} i m_{7} K_{m n}{ }^{[I J} K^{n K L]}\left(u_{i j}{ }^{I J} u_{k l}{ }^{K L}-v_{i j I J} v_{k l K L}\right) \\
& +\frac{4}{7} i m_{7} K_{m}{ }^{I J}\left(v_{i j M J} u_{k l}{ }^{M I}-u_{i j}{ }^{M J} v_{k l M I}\right)
\end{aligned}
$$

However, since our identities, e.g. (3.15), are written in terms of the usual $S^{7}$ covariant derivative $\stackrel{\circ}{D}_{m}$, we use this connection for convenience.

From eqs. (3.28) and (3.35), we can now see exactly how the solution given in equation (3.19) of ref. [3] for $\mathcal{P}_{m i j k l}$ is modified by the generalised connection coefficients. It is clear from eq. (3.28) that the role of the generalised connection term is to fully antisymmetrise $\stackrel{\circ}{D}_{m} A_{n p q}$ and $\stackrel{\circ}{D}_{m_{1}} A_{m_{2} \ldots m_{7}}$ terms coming from $\stackrel{\circ}{D}_{m} \mathcal{V}_{\mathcal{M}}$. This gives the field strength components $F_{m n p q}$ and $F_{m_{1} \ldots m_{7}}$ in $\mathcal{P}_{m i j k l}$ - without the generalised vielbein postulate this task would be an unwieldy problem.

\footnotetext{
${ }^{7}$ In ref. [3], $\mathcal{P}_{m i j k l}$ is denoted by $\mathcal{A}_{m i j k l}$.
} 
We now make use of eq. (3.34), remembering that the contributions from the generalised connections vanish, and insert the explicit formulae for the vielbein components, (3.11)-(3.14), into the expression for the Freund-Rubin term, (3.29). Defining

$$
\begin{aligned}
& \mathrm{X}_{r s}{ }^{i j k l}(x, y)=K^{I J K L}(y)\left(u_{r s}^{I M}+v_{r s I M}\right)\left(u_{[J K}^{i j} u_{L M]}^{k l}-v^{i j[J K} v^{k l L M]}\right)(x), \\
& \mathrm{Y}_{r s}{ }^{i j k l}(x, y)=K_{m}{ }^{K L} K_{m}^{K L}(y)\left(u_{r s}^{I J}+v_{r s I J}\right)\left(u_{K M}^{i j} v^{k l L M}-v^{i j K M} u_{L M}^{k l}\right)(x),
\end{aligned}
$$

where $K^{I J K L}(y)=K_{m}^{[I J}(y) K^{m K L]}(y)$, we find that

$$
\begin{aligned}
\mathcal{V}^{m 8}{ }^{r s} \mathcal{V}^{\mathcal{N}}{ }_{k l} \stackrel{\circ}{D}_{m} \mathcal{V}_{\mathcal{N} i j} & =-\frac{\sqrt{2} m_{7}}{28}\left(3 \mathrm{X}^{r s}{ }_{i j k l}-2 \mathrm{Y}^{r s}{ }_{i j k l}\right) \\
\mathcal{V}^{p 8}{ }_{r s} \mathcal{V}_{p q}{ }^{[i j} \mathcal{V}^{q 8 k l]} & =\frac{\sqrt{2}}{64}\left(2 \mathrm{X}_{r s}{ }^{i j k l}+\mathrm{Y}_{r s}{ }^{i j k l}\right) .
\end{aligned}
$$

Thus, the Freund-Rubin term is

$$
\mathfrak{f}_{F R}(x, y)=\frac{m_{7}}{168 \sqrt{2}}\left(3 \mathrm{X}^{r s}{ }_{i j k l}-2 \mathrm{Y}^{r s}{ }_{i j k l}\right)\left(2 \mathrm{X}_{r s}{ }^{i j k l}+\mathrm{Y}_{r s}{ }^{i j k l}\right)(x, y) .
$$

\section{Examples}

In the following, we evaluate the Freund-Rubin term (3.40) for the $\mathrm{G}_{2}$ invariant sector $[37,38]$. We refer the reader to appendices $\mathrm{B}$ and $\mathrm{C}$ for the Freund-Rubin term for the $\mathrm{SO}(3) \times \mathrm{SO}(3)$ and $\mathrm{SU}(4)^{-}$invariant sectors. At stationary points, $\mathfrak{f}_{F R}$ is proportional to the scalar potential. This has already been noted in ref. [21]. Eq. (3.40) now gives a general expression for $\mathfrak{f}_{F R}$ away from stationary points. In the following examples, we will see that this expression always consists of two parts: the first part is proportional to the scalar potential $V$ - this has been verified for many stationary points [21]. The second part is proportional to a variation of the potential and depends on internal coordinates. Thus, the Freund-Rubin term is only constant at stationary points. In uplifts of flows the Freund-Rubin term will, in general, be both $x$ and $y$-dependent.

\subsection{Freund-Rubin term in the $\mathrm{G}_{2}$ invariant sector}

In a 'unitary gauge,' the 56-bein takes the special form

$$
\mathcal{V}=\left(\begin{array}{ll}
u_{I J}{ }^{K L} & v_{I J K L} \\
v^{I J K L} & u^{I J}{ }_{K L}
\end{array}\right)=\exp \left(\begin{array}{cc}
0 & \phi_{I J K L} \\
\phi^{I J K L} & 0
\end{array}\right)
$$

For the $\mathrm{G}_{2}$ invariant sector

$$
\phi_{I J K L} \equiv \phi_{I J K L}(\alpha, \lambda)=\frac{\lambda}{2}\left(\cos \alpha C_{+}^{I J K L}+i \sin \alpha C_{-}^{I J K L}\right)
$$

with the $\mathrm{SO}(7)^{+}$and $\mathrm{SO}(7)^{-}$invariant 4 -form tensors $C_{+}^{I J K L}$ and $C_{-}^{I J K L}$, respectively. The common invariance group is $\mathrm{G}_{2}=\mathrm{SO}(7)^{+} \cap \mathrm{SO}(7)^{-}$.

The scalar potential for the $\mathrm{G}_{2}$ invariant sector, calculated from eq. (6.3), reads

$$
V(\alpha, \lambda)=2 g^{2}\left[\left(7 v^{4}-7 v^{2}+3\right) c^{3} s^{4}+\left(4 v^{2}-7\right) v^{5} s^{7}+c^{5} s^{2}+7 v^{3} c^{2} s^{5}-3 c^{3}\right]
$$




$$
\begin{aligned}
=2(c+v s)^{2}\left(7 v^{3} s^{3}+4 v^{5} s^{5}-\right. & 14 c v^{2} s^{2}-8 c v^{4} s^{4}+14 c^{2} v s \\
+ & \left.5 c^{2} v^{3} s^{3}-7 c^{3}+5 c^{3} v^{2} s^{2}-8 c^{4} v s+4 c^{5}\right) .
\end{aligned}
$$

Here, $g$ is the gauge coupling constant and

$$
c=\cosh 2 \lambda, \quad s=\sinh 2 \lambda, \quad v=\cos \alpha .
$$

Taking the derivative of the potential with respect to $\alpha$ and $\lambda$ yields

$$
\begin{aligned}
\frac{\mathrm{d} V}{\mathrm{~d} \alpha}= & -14 v s^{2} \sin \alpha(c+v s)\left(5 v^{2} s^{2}+4 v^{4} s^{4}-5 c v s-4 c v^{3} s^{3}+2 c^{2}-c^{2} v^{2} s^{2}+5 c^{3} v s-2 c^{4}\right), \\
\frac{\mathrm{d} V}{\mathrm{~d} \lambda}= & 28 \frac{c}{s}(c+v s)\left(2 v^{2} s^{2}+7 v^{4} s^{4}+4 v^{6} s^{6}-5 c v s-10 c v^{3} s^{3}-4 c v^{5} s^{5}+5 c^{2}\right. \\
& \left.+c^{2} v^{2} s^{2}-3 c^{2} v^{4} s^{4}+9 c^{3} v s+10 c^{3} v^{3} s^{3}-9 c^{4}-3 c^{4} v^{2} s^{2}-4 c^{5} v s+4 c^{6}\right) .
\end{aligned}
$$

We write the $u$ and $v$ tensors in the following basis of $\mathrm{G}_{2}$ invariants [4]

$$
\delta_{K L}^{I J}, \quad C_{+}^{I J K L}, \quad C_{-}^{I J K L}, \quad D_{ \pm}=\frac{1}{2}\left(C_{+}^{I J M N} C_{-}^{M N K L} \pm C_{-}^{I J M N} C_{+}^{M N K L}\right) .
$$

Here, $C_{+}^{I J K L}$ is selfdual and $C_{-}^{I J K L}$ is anti-selfdual. Having chosen a symmetric gauge for the $d=4$ 56-bein, we do not distinguish between $\mathrm{SU}(8)$ and $\mathrm{SO}(8)$ indices. We find $[4,15]$

$$
\begin{gathered}
u_{I J}^{K L}(\lambda, \alpha)=p^{3} \delta_{I J}^{K L}+\frac{1}{2} p q^{2} \cos ^{2} \alpha C_{+}^{I J K L}-\frac{1}{2} p q^{2} \sin ^{2} \alpha C_{-}^{I J K L}-\frac{1}{8} i p q^{2} \sin 2 \alpha D_{-}^{I J K L}, \\
v_{I J K L}(\lambda, \alpha)=q^{3}\left(\cos ^{3} \alpha-i \sin ^{3} \alpha\right) \delta_{K L}^{I J}+\frac{1}{2} p^{2} q \cos \alpha C_{+}^{I J K L}+\frac{1}{2} i p^{2} q \sin \alpha C_{-}^{I J K L} \\
-\frac{1}{8} q^{3} \sin 2 \alpha(\sin \alpha-i \cos \alpha) D_{+}^{I J K L}
\end{gathered}
$$

The $x$-dependence is kept in $\lambda=\lambda(x)$ through

$$
p=\cosh \lambda, \quad q=\sinh \lambda .
$$

$u_{K L}^{I J}$ and $v^{I J K L}$ are obtained from the above equations by complex conjugation.

Plugging the explicit form of the $u$ and $v$ tensors into the expression of the FreundRubin term and identifying

$$
\xi(y)=-\frac{1}{16} C_{+}^{I J K L} K_{m}{ }^{I J} K^{m K L},
$$

we find the Freund-Rubin term in the $\mathrm{G}_{2}$ invariant sector:

$$
\begin{aligned}
\mathfrak{f}_{F R}=-\sqrt{2} m_{7}(c+v s)^{2}\left(7 v^{3} s^{3}+\right. & 4 v^{5} s^{5}-14 c v^{2} s^{2}-8 c v^{4} s^{4}+14 c^{2} v s \\
& \left.+5 c^{2} v^{3} s^{3}-7 c^{3}+5 c^{3} v^{2} s^{2}-8 c^{4} v s+4 c^{5}\right)
\end{aligned}
$$




$$
+\frac{\sqrt{2}}{3} m_{7} \xi(c+v s)^{2} c v s\left(3 v s+2 v^{3} s^{3}-3 c-c v^{2} s^{2}-c^{2} v s+2 c^{3}\right) .
$$

While the first two lines are $y$-independent, all the $y$-dependence here is contained in the factor $\xi(y)$ in the last line. Using eqs. (4.3), (4.5) and (4.6), the above expression can be rewritten as

$$
\mathfrak{f}_{F R}=\frac{m_{7}}{\sqrt{2} g^{2}}\left(-V+\frac{\xi}{21 s}\left(\frac{s \cos \alpha}{2} \frac{\mathrm{d} V}{\mathrm{~d} \lambda}-c \sin \alpha \frac{\mathrm{d} V}{\mathrm{~d} \alpha}\right)\right) .
$$

This result is exactly of the expected form. The term proportional to the scalar potential is coordinate invariant. All other terms are proportional to the derivatives of $V$ with respect to $\alpha$ and $\lambda$ and thus vanish at the stationary points, that is, when the equations of motion are obeyed. Off-shell, there is a linear dependence on $\xi(y)$ so the extra terms do depend on internal coordinates. Furthermore, $\mathfrak{f}_{F R}$ is $x$-dependent via $s, c$ and $\alpha$. Note that the $\mathrm{G}_{2}$ invariant sector also includes as special cases the $\mathrm{SO}(7)^{ \pm}$invariant sectors for appropriate values of $\alpha$ :

$$
\mathfrak{f}_{F R}=\left\{\begin{array}{ll}
\left.\frac{m_{7}}{\sqrt{2} g^{2}}\left(-V+\frac{\xi}{42} \frac{\mathrm{d} V}{\mathrm{~d} \lambda}\right)\right|_{v=1} & \mathrm{SO}(7)^{+} \\
-\left.\frac{m_{7}}{\sqrt{2} g^{2}} V\right|_{v=0} & \mathrm{SO}(7)^{-}
\end{array} .\right.
$$

(recall that $d V / d \alpha$ vanishes for $v=0$ ).

We repeat this calculation in appendices $\mathrm{B}$ and $\mathrm{C}$ for the $\mathrm{SO}(3) \times \mathrm{SO}(3)$ and $\mathrm{SU}(4)^{-}$ invariant sectors and find expressions similar to eqs. (4.13) and (4.14). Motivated by these results we state a general conjecture for the Freund-Rubin term in section 6 .

\section{Ansätze for other components of the 4-form field strength}

Given the new ansatz for the Freund-Rubin term, a natural question that arises is whether similar ansätze for the other components of the 4-form field strength can also be teased out of the generalised vielbein postulates. The generalised spin connection and non-metricity from eqs. (3.23) and (3.24) in the internal GVP depend on $F_{m n p q}$ as well as $\mathfrak{f}_{F R}$. Therefore, one can also project onto the component giving $F_{m n p q}$. Indeed this is done in refs. $[3,6]$ using only the original generalised vielbein $e_{A B}^{m}$. However, we use the full 56-bein and its various components and take account of the generalised connection term. We can project onto the $F_{m n p q}$ term by performing the following contraction of $\mathcal{P}_{m A B C D}$ with components of the 56-bein:

$$
\mathcal{P}_{m A B C D}\left(\mathcal{V}^{r 8 A B} \mathcal{V}_{p q}^{C D}+\frac{1}{4 !} \epsilon^{A B C D E F G H} \mathcal{V}_{E F}^{r 8} \mathcal{V}_{p q G H}\right)=\frac{1}{16} \Delta^{-1} g^{r n} F_{m n p q}
$$

Therefore, from eq. (3.25), we find that the uplift ansatz for $F_{m n p q}$ is given by

$$
\Delta^{-1} g^{n r} F_{m n p q}=-16 i\left(\mathcal{V}^{\mathcal{N}}{ }_{i j} \partial_{m} \mathcal{V}_{\mathcal{N} k l}-\Gamma_{m \mathcal{N}^{\mathcal{P}}} \mathcal{V}^{\mathcal{N}}{ }_{i j} \mathcal{V}_{\mathcal{P} k l}\right) \mathcal{V}^{r 8 i j} \mathcal{V}_{p q}{ }^{k l}+h . c \ldots
$$

The ansatz above is not as direct as the formula for the Freund-Rubin term (3.27). Firstly, as with the non-linear flux ansatz [5] one needs to invert the metric to obtain 
$F_{m n p q}{ }^{8}$ Moreover, the contributions from the generalised connection components do not vanish. It is these terms that antisymmetrise the $\partial A$ terms in $\partial \mathcal{V}$ to give the field strength. Hence without these terms the field strength components would not be fully antisymmetric - a point that was noted in ref. [21]. We therefore conclude that differentiating $A_{m n p}$ obtained from the non-linear uplift flux ansatz is a simpler way of finding the internal components of $F_{m n p q}$ than the ansatz derived from the internal GVP, see eq. (5.2).

While the generalised spin connection and non-metricity are parametrised by $F_{m n p q}$ and $\mathfrak{f}_{F R}$, the connections of the external GVP [1] are given in terms of the $F_{\mu \nu \rho m}$ and $F_{\mu m n p}$ components of the 4 -form field strength. In $\mathrm{E}_{7(7)}$ covariant form, the external GVP is [36]

$$
\partial_{\mu} \mathcal{V}_{\mathcal{M} A B}+2 \hat{\mathcal{L}}_{\mathcal{B}_{\mu}} \mathcal{V}_{\mathcal{M} A B}+\mathcal{Q}_{\mu[A}^{C} \mathcal{V}_{\mathcal{M} B] C}=\mathcal{P}_{\mu A B C D} \mathcal{V}_{\mathcal{M}}{ }^{C D}
$$

where $\hat{\mathcal{L}}$ is the $\mathrm{E}_{7(7)}$ generalised Lie derivative $[24,39]^{9}$

$$
\hat{\mathcal{L}}_{\Lambda} X_{\mathcal{M}}=\Lambda^{m} \partial_{m} X_{\mathcal{M}}+12\left(t^{\boldsymbol{\alpha}}\right)_{\mathcal{M}}{ }^{\mathcal{N}}\left(t_{\boldsymbol{\alpha}}\right)_{\mathcal{P}}{ }^{q 8} \partial_{q} \Lambda^{\mathcal{P}} X_{\mathcal{N}}
$$

and the connection coefficients are of the form

$$
\begin{gathered}
\mathcal{Q}_{\mu B}^{A}=-\frac{1}{2}\left[e^{m}{ }_{a} D_{m} B_{\mu}{ }^{n} e_{n b}-\left(e^{p}{ }_{a} \mathcal{D}_{\mu} e_{p b}\right)\right] \Gamma_{A B}^{a b}-\frac{\sqrt{2}}{12} e_{\mu}{ }^{\alpha}\left(F_{\alpha a b c} \Gamma_{A B}^{a b c}-\eta_{\alpha \beta \gamma \delta} F^{\beta \gamma \delta a} \Gamma_{a A B}\right), \\
\mathcal{P}_{\mu A B C D}=\frac{3}{4}\left[e^{m}{ }_{a} D_{m} B_{\mu}{ }^{n} e_{n b}-\left(e^{p}{ }_{a} \mathcal{D}_{\mu} e_{p b}\right)\right] \\
\Gamma_{[A B}^{a} \Gamma_{C D]}^{b}-\frac{\sqrt{2}}{8} e_{\mu}{ }^{\alpha} F_{a b c \alpha} \Gamma_{[A B}^{a} \Gamma_{C D]}^{b c} \\
-\frac{\sqrt{2}}{48} e_{\mu \alpha} \eta^{\alpha \beta \gamma \delta} F_{a \beta \gamma \delta} \Gamma_{b[A B} \Gamma_{C D]}^{a b},
\end{gathered}
$$

where

$$
\mathcal{D}_{\mu} \equiv \partial_{\mu}-B_{\mu}^{m} D_{m}
$$

We recall that $D_{m}$ is the covariant derivative with respective to the connection $\Gamma_{m n}^{p}$ and $e_{\mu}{ }^{\alpha}$ is the vierbein.

Given a particular reduction ansatz, the external GVP (5.3) reduces to the Cartan equation of the scalars of the four-dimensional maximal gauge theory [12]:

$$
\partial_{\mu} \hat{\mathcal{V}}_{\mathcal{M} i j}-g A_{\mu}{ }^{\mathcal{P}} X_{\mathcal{P} \mathcal{M}}{ }^{\mathcal{N}} \hat{\mathcal{V}}_{\mathcal{N} i j}+\mathcal{Q}_{\mu[i}^{k} \hat{\mathcal{V}}_{\mathcal{M} j] k}=\mathcal{P}_{\mu i j k l} \hat{\mathcal{V}}_{\mathcal{M}}^{k l}
$$

where $\hat{\mathcal{V}}$ is given in eq. (3.10) and $X_{\mathcal{M}}$ are generators of the gauge algebra and are related to the embedding tensor $\Theta_{\mathcal{M}}{ }^{\alpha}$ as follows

$$
X_{\mathcal{M}}=\Theta_{\mathcal{M}}{ }^{\alpha} t_{\alpha}
$$

\footnotetext{
${ }^{8}$ In fact, contracting $\mathcal{P}_{m A B C D}$ with other components of the 56-bein would directly give an ansatz for $F_{m n p q}$ without need to invert the metric. However, this leads to a more complicated expression involving $A_{m n p}$ and $A_{m_{1} \ldots m_{6}}$ contributions on the right-hand side.

${ }^{9}$ The generalised Lie derivative encodes the diffeomorphisms and gauge transformations of the $D=11$ fields $[24,36]$. In approaches where the base space is also enlarged, e.g. ref. [35, 39], the partial derivatives also carry $\mathrm{E}_{7(7)}$ indices.
} 
The embedding tensor projects out at most 28 of the 56 vectors $A_{\mu}^{\mathcal{P}}$ [12]. The $\mathcal{Q}_{\mu j}^{i}$ are related to $\mathcal{Q}_{\mu B}^{A}$ by an inhomogeneous relation, while $\mathcal{P}_{\mu i j k l}$ are covariantly related to $\mathcal{P}_{\mu A B C D}$ via the eight Killing spinors of the vacuum solution of the maximal gauged supergravity.

Let us consider the term proportional to $F_{\mu \nu \rho m}$ in $\mathcal{P}_{\mu A B C D}$. This term can be projected out as follows:

$$
\mathcal{P}_{\mu A B C D} \mathcal{V}^{n 8 A B} \mathcal{V}_{m n}{ }^{C D}=\frac{\sqrt{2}}{8} e_{\mu \delta} \eta^{\alpha \beta \gamma \delta} F_{m \alpha \beta \gamma}
$$

Thus, we obtain the uplift ansatz

$$
F_{\mu \nu \rho m}=\frac{2 \sqrt{2}}{3} i \eta_{\mu \nu \rho}{ }^{\sigma}\left(\hat{\mathcal{V}}^{\mathcal{M}}{ }_{i j} \partial_{\sigma} \hat{\mathcal{V}}_{\mathcal{M} k l}-g A_{\sigma}{ }^{\mathcal{P}} X_{\mathcal{P} \mathcal{M}}{ }^{\mathcal{N}} \hat{\mathcal{V}}^{\mathcal{M}}{ }_{i j} \hat{\mathcal{V}}_{\mathcal{N} k l}\right) \mathcal{V}^{n 8 i j} \mathcal{V}_{m n}{ }^{k l}
$$

This provides a non-linear ansatz for $F_{\mu \nu \rho m}$ for any truncation of $D=11$ supergravity to four dimensions. Note that the ansätze for $\mathcal{V}^{n 8}$ and $\mathcal{V}_{m n}$ will be linear and follow directly from the linear ansätze for the vectors.

In the $S^{7}$ truncation, the connections in eq. (5.3) and (5.8) are related via the eight Killing spinors $\eta^{i}$ on the $S^{7}[3]$

$$
\begin{aligned}
\mathcal{Q}_{\mu j}^{i} & =\eta_{A}^{i} \eta_{j}^{B}\left(\mathcal{Q}_{\mu B}^{A}-\frac{\sqrt{2} i}{4} m_{7} A_{\mu}{ }^{K L} K^{n K L} \stackrel{\circ}{e}_{n}{ }^{a} \Gamma_{a}{ }^{A} B\right), \\
\mathcal{P}_{\mu i j k l} & =\eta_{i}^{A} \eta_{j}^{B} \eta_{k}^{C} \eta_{l}^{D} \mathcal{P}_{\mu A B C D},
\end{aligned}
$$

where $A_{\mu}{ }^{K L}$ are the 28 vectors of the $d=4$ theory that are gauged. The generators of the gauge algebra are given by [16]

$$
X_{\mathcal{M N}} \mathcal{P}=\left\{\begin{array}{l}
X_{I J K L}{ }^{M N}=X_{I J}{ }^{K L}{ }_{M N}=2 \delta_{I J}^{R[K} \delta_{M N}^{L] R} \\
0 \quad \text { otherwise }
\end{array}\right.
$$

and the reduction ansatz for the relevant components of the 56-bein are given in eqs. (3.11) and (3.12). With these substitutions, eq. (5.11) reduces to

$$
\begin{aligned}
F_{\mu \nu \rho m}= & -\frac{\sqrt{2}}{48} \eta_{\mu \nu \rho}{ }^{\sigma} K^{n I J} K_{m n}{ }^{K L}\left(u^{i j}{ }_{I J}+v^{i j I J}\right)\left(u_{K L}^{k l}-v^{k l K L}\right) \\
& \times\left(\hat{\mathcal{V}}^{\mathcal{M}}{ }_{i j} \partial_{\sigma} \hat{\mathcal{V}}_{\mathcal{M} k l}-2 \sqrt{2} m_{7} A_{\sigma}{ }^{M N} \hat{\mathcal{V}}^{M P}{ }_{i j} \hat{\mathcal{V}}_{N P k l}-2 \sqrt{2} m_{7} A_{\sigma}{ }^{M N} \hat{\mathcal{V}}^{M P}{ }_{k l} \hat{\mathcal{V}}_{N P i j}\right) .
\end{aligned}
$$

This is the non-linear uplift ansatz for $F_{\mu \nu \rho m}$ for the $S^{7}$ reduction of $D=11$ supergravity. We note, as a check, that in the $\mathrm{SO}(7)^{+}$sector the above expression reproduces the correct result, viz.

$$
F_{\mu \nu \rho m}=\frac{\sqrt{2}}{6} i \eta_{\mu \nu \rho} \sigma \partial_{\sigma} \lambda \partial_{m} \xi
$$

The above ansatz for $F_{\mu \nu \rho m},(5.11)$, provides a considerable simplification over computing the Hodge dual of $F_{\mu m_{1} \ldots m_{6}}$ calculated using the ansatz for the metric, 3-form and 6 -form. This is clear even in the relatively simple case of the $\mathrm{SO}(7)^{+}$sector. The advantage of the ansätze (5.11) for $F_{\mu \nu \rho m}$ (and its specialisation to the $S^{7}$ reduction (5.15)) 
and (3.40) for the Freund-Rubin term is that they do not require differentiation or the metric to be inverted.

The connection $\mathcal{P}_{\mu A B C D}$ also depends on the $F_{\mu m n p}$ components of the field strength. However, as is the case with the ansatz for $F_{m n p q},(5.2)$, we do not obtain a direct ansatz. Therefore, for the $F_{\mu m n p}$ and $F_{m n p q}$ components the GVPs do not provide more efficient ansätze. However, these components are easily calculated using the 3 -form ansatz [5]. We are fortunate that the GVPs give direct ansätze for the components of the field strength that are otherwise difficult to calculate.

The only remaining component of the field strength that we have not thus far discussed is the $F_{\mu \nu m n}$ components, which does not feature in the GVPs. However, this component does enter the fermion supersymmetry transformations via

$$
\mathcal{G}_{\alpha \beta A B} \equiv-\frac{1}{8} i \Delta^{-1 / 2} e_{[\alpha}{ }^{\mu} e_{\beta]}{ }^{\nu} \mathcal{D}_{\mu} B_{\nu}{ }^{n} \Gamma_{n A B}+\frac{\sqrt{2}}{32} i \Delta^{-1 / 2} F_{\alpha \beta m n} \Gamma_{A B}^{m n}
$$

Comparing the fermion supersymmetry transformations in four $[12,16]$ and eleven dimensions [6], we make the following identification

$$
\mathcal{H}_{\alpha \beta i j}=4 \sqrt{2} \eta_{i}^{A} \eta_{j}^{B} \mathcal{G}_{\alpha \beta A B},
$$

where $\mathcal{H}_{\alpha \beta i j}$ is related to the covariantised field strength $\mathcal{G}_{\alpha \beta}{ }^{\mathcal{M}}[12]$

$$
\mathcal{H}_{\alpha \beta i j}=\hat{\mathcal{V}}_{\mathcal{M} i j} \mathcal{G}_{\alpha \beta}{ }^{\mathcal{M}}
$$

Contracting eq. (5.17) with $\mathcal{V}_{m n}{ }^{A B}$ gives an imaginary expression

$$
\mathcal{V}_{m n}{ }^{A B} \mathcal{G}_{\alpha \beta A B}=-\frac{1}{8} i \Delta^{-1} F_{\alpha \beta m n}+\frac{3}{2} i \Delta^{-1} A_{m n p}\left(e^{\mu}{ }_{[\alpha} e^{\nu}{ }_{\beta]} \mathcal{D}_{\mu} B_{\nu}{ }^{p}-g^{p q} e^{\mu}{ }_{[\alpha} \partial_{q} e_{\mu \beta]}\right) .
$$

Using eqs. (5.18), (5.19) and the above equation, we obtain the non-linear uplift ansatz for $F_{\mu \nu m n}$ for any reduction

$$
F_{\mu \nu m n}=\sqrt{2} i \mathcal{V}_{m n}{ }^{i j} \hat{\mathcal{V}}_{\mathcal{M} i j} \mathcal{G}_{\mu \nu}{ }^{\mathcal{M}}+\frac{3}{2} A_{m n p}\left(\mathcal{D}_{[\mu} B_{\nu]}^{p}+g^{p q} e_{[\mu}{ }^{\alpha} \partial_{q} e_{\nu] \alpha}\right)
$$

Specialising to the $S^{7}$ reduction gives

$$
F_{\mu \nu m n}=\frac{\sqrt{2}}{8}\left(K_{m n}{ }^{I J} \mathcal{G}_{\mu \nu I J}-12 \Delta^{-1} A_{m n p} K^{p}{ }_{I J} \mathcal{H}_{\mu \nu}{ }^{I J}\right)
$$

where [12]

$$
\mathcal{G}_{\mu \nu}{ }^{\mathcal{M}}=\left(\begin{array}{l}
\mathcal{H}_{\mu \nu}^{I J} \\
\mathcal{G}_{\mu \nu I J}
\end{array}\right) .
$$

Hence, $F_{\mu \nu m n}$ is only non-trivial for four-dimensional solutions with non-zero vector expectation values. 


\section{General form of the Freund-Rubin term}

\subsection{The conjecture}

We observed in section 4 and appendices B and C that for various examples the FreundRubin term is proportional to the potential, with the constant of proportionality given by $-m_{7} /\left(\sqrt{2} g^{2}\right)[21]$, and a $y$-dependent part that contains variations of the potential. Furthermore, the $y$-dependence only enters linearly via the invariant scalars ( $\xi$ in $\mathrm{G}_{2}$ and $(\xi, \zeta)$ in $\mathrm{SO}(3) \times \mathrm{SO}(3)$, see appendix $\mathrm{B})$. In particular, if the sector under consideration does not contain an invariant scalar (such as $\mathrm{SO}(7)^{-}$or $\mathrm{SU}(4)^{-}$), then $\mathfrak{f}_{F R}$ is $y$-independent and proportional to the potential. In the following, we will state a general conjecture for the Freund-Rubin term that respects all these observations.

First, we state the general expressions for the potential $V$ and its variation $\delta V$ in terms of the tensors $u_{i j}{ }^{I J}$ and $v_{i j} I J$. We define the $T$-tensor [16]

$$
T_{i}^{j k l}=\left(u_{I J}^{k l}+v^{k l I J}\right)\left(u_{i m}{ }^{J K} u_{K I}^{j m}-v_{i m J K} v^{j m K I}\right)
$$

and its components

$$
A_{1}^{i j}=\frac{4}{21} T_{k}{ }^{i k j}, \quad A_{2}{ }^{j k l}=-\frac{4}{3} T_{i}{ }^{[j k l]} .
$$

In terms of the above tensors the potential is given by [16]

$$
V=\frac{1}{24} g^{2} A_{2}{ }^{j k l} A_{2}{ }^{i}{ }_{j k l}-\frac{3}{4} g^{2} A_{1}^{i j} A_{1 i j} .
$$

In order to determine the variation of the potential, we consider an infinitesimal $\mathrm{E}_{7(7)}$ variation of the 56-bein of the form [25]

$$
\delta \mathcal{V}=-\frac{\sqrt{2}}{4}\left(\begin{array}{cc}
0 & \Sigma^{i j k l} \\
\Sigma_{i j k l} & 0
\end{array}\right) \mathcal{V},
$$

where $\Sigma$ is complex selfdual. Given the variation of the 56 -bein given above, to first order, the potential varies as [25]

$$
\delta V=\frac{\sqrt{2}}{24} g^{2} Q^{i j k l} \Sigma_{i j k l}+\text { h.c. }
$$

where the $Q$-tensor is

$$
Q^{i j k l}=\frac{3}{4} A_{2 m}{ }^{n[i j} A_{2 n}{ }^{k l] m}-A_{1}{ }^{m[i} A_{2} m^{j k l]} .
$$

Since, the expression on the right-hand side of eq. (6.5) gives the variation of the potential to first order, it must vanish at the stationary points. In particular, since $\Sigma_{i j k l}$ is an arbitrary complex selfdual tensor, $Q^{i j k l}$ is complex anti-selfdual at stationary points.

We define a complex selfdual combination of $u$ and $v$ tensors

$$
\hat{\Sigma}_{i j k l}(x, y) \equiv\left(u_{i j}^{I J}(x) u_{k l}^{K L}(x)-v_{i j I J}(x) v_{k l K L}(x)\right) K^{I J K L}(y),
$$


where we have written out the coordinate dependence explicitly so as to make the dependence of $\hat{\Sigma}$ on all eleven coordinates clear. Making use of the $Q$-tensor, we are now able to formulate a conjecture for the Freund-Rubin term:

$$
\mathfrak{f}_{F R}=-\frac{m_{7}}{\sqrt{2} g^{2}}\left(V-\frac{g^{2}}{24}\left(Q^{i j k l} \hat{\Sigma}_{i j k l}+\text { h.c. }\right)\right) \text {. }
$$

The second term on the right-hand side is inevitably $y$-dependent, and it vanishes when $Q^{i j k l}$ is complex anti-selfdual, which is precisely the minimisation condition for the potential.

To prove this formula, one has to manipulate eq. (3.40) using $\mathrm{E}_{7(7)}$ identities for the $u$ and $v$ tensors $[3,16]$. However, the proof will also probably require identities derived from the quartic invariant (see, e.g. ref. [14]). We leave this proof (which is probably even more complicated than the one given in ref. [3] for the $y$-independence of the $A_{1}$ and $A_{2}$ tensors coming from the $S^{7}$ truncation) for future work. In the remainder of this section, we will prove the conjecture up to quadratic order and verify it for the $\mathrm{G}_{2}$ invariant sector.

\subsection{Proof of the conjecture up to quadratic order}

In this section, we prove the equality of eqs. (3.40) and (6.8) for a perturbative expansion of the $u$ and $v$ tensors. As in eq. (4.1) we use the unitary gauge,

$$
\mathcal{V}=\exp \left(\begin{array}{cc}
0 & \phi_{I J K L} \\
\phi^{I J K L} & 0
\end{array}\right)
$$

where we do not need to distinguish between $\mathrm{SU}(8)$ and $\mathrm{SO}(8)$ indices. Thus,

$$
u_{I J}{ }^{K L}=(\cosh \phi)_{I J}{ }^{K L}, \quad v_{I J K L}=(\sinh \phi)_{I J K L} .
$$

Here, we denote

$$
\left(\phi^{0}\right)_{I J}{ }^{K L}=\delta_{I J}{ }^{K L}, \quad\left(\phi^{2}\right)_{I J}{ }^{K L}=\phi_{I J M N} \phi^{M N K L} .
$$

Complex conjugation is realised by raising and lowering indices. Furthermore, the potential is complex selfdual,

$$
\phi_{I J K L}^{*}=\phi^{I J K L}=\frac{1}{24} \epsilon^{I J K L M N P Q} \phi_{M N P Q}
$$

Up to quadratic order, we obtain

$$
u_{I J}{ }^{K L}=\delta_{I J}^{K L}+\frac{1}{2} \phi_{I J M N} \phi^{M N K L}+\mathcal{O}\left(\phi^{4}\right), \quad v_{I J K L}=\phi_{I J K L}+\mathcal{O}\left(\phi^{3}\right) .
$$

Substituting the expansions for the $u$ and $v$ tensors in the expressions for $\mathrm{X}_{r s}{ }^{i j k l}$ and $\mathrm{Y}_{r s}{ }^{i j k l},(3.36)$ and $(3.37)$, we find up to terms $\mathcal{O}\left(\phi^{2}\right)$,

$$
\begin{aligned}
\mathrm{X}_{r s}{ }^{i j k l} \mathrm{X}^{r s}{ }_{i j k l}= & 168+19 \phi^{I J K L} \phi_{I J K L}-\phi_{I J K L} \phi_{I J K L} \\
& +3 K^{I J K L}\left(2 \phi_{I J K L}+3 \phi^{I J M N} \phi_{M N K L}\right)+\frac{1}{24}\left(K^{I J K L} \phi_{I J K L}\right)^{2},
\end{aligned}
$$




$$
\begin{aligned}
\mathrm{X}_{r s}{ }^{i j k l} \mathrm{Y}^{r s}{ }_{i j k l}= & -6 \phi^{I J K L} \phi_{I J K L}-6 \phi_{I J K L} \phi_{I J K L} \\
& +2 K^{I J K L}\left(4 \phi_{I J K L}+3 \phi^{I J M N} \phi_{M N K L}\right)+\frac{1}{4}\left(K^{I J K L} \phi_{I J K L}\right)^{2}, \\
\mathrm{Y}_{r s}{ }^{i j k l} \mathrm{Y}^{r s}{ }_{i j k l}= & 32 \phi^{I J K L} \phi_{I J K L}+24 K^{I J K L} \phi^{I J M N} \phi_{M N K L},
\end{aligned}
$$

where now all the $y$-dependence is contained in $K^{I J K L}(y)$. In deriving the above expressions, we make use of the following identities

$$
\begin{aligned}
K^{I J K P} K_{L M N P} & =6 \delta_{L M N}^{I J K}+9 \delta_{[L}^{[I} K^{J K]}{ }_{M N]}, \\
K^{[I J K L} K^{M] N P Q} & \left.=\frac{1}{5} \epsilon^{I J K L M N P Q}+12 K^{[I J K}{ }_{[N} \delta^{L}{ }_{P} \delta^{M]} Q\right], \\
K^{m I J} K^{n K L} K_{m n}{ }^{M N} & =8 \delta^{[I}{ }_{[K} \delta^{J][M} \delta^{N]}{ }_{L]}+4 \delta^{[M}{ }_{[I} K^{N]}{ }_{J] K L}+4 \delta_{\left[{ }_{[M} K^{L]}{ }_{N] I J}-4 \delta_{[K}^{[I} K^{J]}\right.}{ }_{L] M N} .
\end{aligned}
$$

It is now straightforward to show that, up to quadratic order, the Freund-Rubin term, (3.40), is

$$
\mathfrak{f}_{F R}=\sqrt{2} m_{7}\left(3+\frac{1}{12} \phi_{I J K L} K^{I J K L}+\frac{1}{6} \phi_{I J K L} \phi^{I J K L}\right)+\mathcal{O}\left(\phi^{3}\right) .
$$

We also find that

$$
V / g^{2}=-6-\frac{1}{3} \phi_{I J K L} \phi^{I J K L}+\mathcal{O}\left(\phi^{3}\right), \quad Q^{i j k l} \hat{\Sigma}_{i j k l}=2 \phi_{I J K L} K^{I J K L}+\mathcal{O}\left(\phi^{3}\right) .
$$

Thus it is easy to verify that the conjectured expression, (6.8), reproduces the expression for the Freund-Rubin term up to quadratic order in the scalar expectation values.

\subsection{Testing the conjecture in the $G_{2}$ invariant sector}

At the stationary points, it has already been established that the conjecture (6.8) holds for the $\mathrm{G}_{2}$ invariant sector [21], see eqs. (4.13) and (4.14). Therefore, it just remains to prove that the $y$-dependent parts of eq. (4.12) and eq. (6.8) coincide, viz.

$$
\left(Q^{i j k l} \hat{\Sigma}_{i j k l}+\text { h.c. }\right)=16 \xi(c+v s)^{2} \operatorname{cvs}\left(3 v s+2 v^{3} s^{3}-3 c-c v^{2} s^{2}-c^{2} v s+2 c^{3}\right),
$$

where again all the $y$-dependence is contained in the factor $\xi(y)$.

Equation (6.6) provides an expression for the $Q$-tensor in terms of the $u$ and $v$ tensors with four free $\mathrm{SU}(8)$ indices. Thus, we can use eqs. (4.8) and (4.9) to write the $Q$-tensor in terms of contracted $\mathrm{G}_{2}$ invariant tensors, (4.7), with four free $\mathrm{SO}(8)$ indices

$$
Q^{i j k l} \rightarrow Q^{I J K L}
$$

In this case, unlike in section 4.1, the $u, v$ tensors are not necessarily contracted over index pairs. However, the resulting expression for $Q^{I J K L}$ must be $\mathrm{G}_{2}$ invariant. Hence, we should be able to write it in the basis given in eq. (4.7). In particular, it is totally antisymmetric, so we must find

$$
Q^{I J K L}=c_{+}(\lambda, \alpha) C_{+}^{I J K L}+c_{-}(\lambda, \alpha) C_{-}^{I J K L}
$$

for some functions $c_{ \pm}$. 
An efficient way to work out the contractions of $\mathrm{SO}(8)$ indices in $Q^{I J K L}$ is to use the $\mathrm{SO}(7)$ decomposition of the $\mathrm{G}_{2}$ invariants (4.7). An $\mathrm{SO}(8)$ index decomposes as $I=(i, 8)$, where $i$ is an $\mathrm{SO}(7)$ index that runs from 1 to 7 . The decomposition of $C_{ \pm}^{I J K L}$ is [25]

$$
C_{ \pm}^{i j k 8}=C^{i j k}, \quad C_{ \pm}^{i j k l}=\mp \frac{1}{6} \eta^{\prime} \epsilon^{i j k l m n p} C_{m n p}
$$

with an arbitrary phase $\eta^{\prime}$. This phase will drop out in our calculations. The $\mathrm{SO}(7)$ tensor $C^{m n p}$ satisfies [25]

$$
C^{[m n p} C^{q] r s}=-\frac{1}{4} \eta^{\prime} \epsilon^{m n p q[r}{ }_{t u} C^{s] t u}, \quad C^{m n r} C_{p q r}=2 \delta_{p q}^{m n}-\frac{1}{6} \eta^{\prime} \epsilon^{m n}{ }_{p q r s t} C^{r s t} .
$$

Moreover, the $D_{-}$-tensor decomposes as follows:

$$
D_{-}^{i j k l}=D_{-}^{i 8 k 8}=0, \quad D_{-}^{i j k 8}=-D_{-}^{k 8 i j}=4 C^{i j k} \quad \Rightarrow \quad D_{-}^{[I J K L]}=0 .
$$

For $D_{+}^{I J K L}$, we find the convenient $\mathrm{SO}(8)$ property

$$
D_{+K L}^{I J}=\frac{2}{3} D_{+}^{M[I} M\left[K \delta^{J]} L\right], \quad \Rightarrow \quad D_{+}^{[I J K L]}=0
$$

so we only need

$$
D_{+}^{M i}{ }_{M j}=-6 \delta_{j}^{i}, \quad D_{+}^{M 8}{ }_{M 8}=42 .
$$

Using all these $\mathrm{SO}(7)$ decompositions together with the identities for the $C$-tensor in eq. (6.26), we find exactly the anticipated form, eq. (6.24) with

$$
\begin{aligned}
c_{+}(\lambda, \alpha)= & \frac{1}{2} c^{3} v s\left(5 c-4 c^{3}+2 s^{2} \sin ^{2} \alpha\right)-\frac{3}{2} c^{2} v^{2} s^{4} \sin ^{2} \alpha-\frac{1}{2} c^{2} v^{3} s^{3}(1-c)(3+7 c) \sin ^{2} \alpha \\
& +\frac{1}{2} v^{4} s^{4}\left(s^{2}(5-3 c) \sin ^{2} \alpha-c-4 c^{3}\right)+2 v^{6} s^{6}(c-1) \sin ^{2} \alpha+\frac{3}{2} i v s^{2}\left(c^{3}+v s^{3}-c^{2}\right) \sin ^{3} \alpha \\
c_{-}(\lambda, \alpha)= & -\frac{3}{2} s^{2}\left(v^{2} c^{3}+v^{3} s^{3}+c^{2} \sin ^{2} \alpha\right) \sin ^{2} \alpha+\frac{1}{2} i c^{4} s \sin \alpha\left(4 s^{2}-1\right) \\
& +\frac{1}{2} i c^{2} v^{2} s^{3}\left(3+2 c-7 c^{2}\right) \sin ^{3} \alpha+\frac{1}{2} i c v^{3} s^{4}\left(5+7 s^{2} \sin ^{2} \alpha-3 c\right) \sin \alpha \\
& -i c^{3} v^{4} s^{3} \sin \alpha+\frac{5}{2} i v^{5} s^{6} \sin \alpha+2 i v^{7} s^{6}(c-1) \sin \alpha .
\end{aligned}
$$

At the $\mathrm{G}_{2}$ invariant stationary point,

$$
c^{2}=\frac{2 \sqrt{3}+3}{5}, \quad s^{2}=\frac{2 \sqrt{3}-2}{5}, \quad v^{2}=\frac{3-\sqrt{3}}{4},
$$

the $Q$-tensor is indeed, complex anti-selfdual because $c_{+}$becomes purely imaginary and $c_{-}$ purely real.

We compute $\hat{\Sigma}_{I J K L} C_{ \pm}^{I J K L}$ using the above $\mathrm{SO}(7)$ decomposition. Identifying,

$$
C_{+}^{I J K L} K^{I J K L}=-16 \xi, \quad C_{-}^{I J K L} K^{I J K L}=0,
$$

we find

$$
\hat{\Sigma}_{I J K L} C_{+}^{I J K L}=-16 \xi\left(c \sin ^{2} \alpha+\cos ^{2} \alpha\right), \quad \hat{\Sigma}_{I J K L} C_{-}^{I J K L}=8 i \xi \sin 2 \alpha(c-1) .
$$

As expected, these expressions are linear in the invariant scalar $\xi$. Eq. (6.22) then follows immediately from eqs. (6.24), (6.30), (6.31) and eq. (6.34). 


\section{Outlook}

In this paper, we derive an explicit formula for the Freund-Rubin term, (3.27), for any consistent truncation of $D=11$ supergravity to four dimensions by means of the internal generalised vielbein postulate [1]. In the case of the $S^{7}$ reduction this reduces to (3.40). Previously, the Freund-Rubin term could be computed using the uplift ansätze for the 6form and 3-form, which involves inverting the metric and differentiating. The new formulae are much simpler. Moreover, for the $S^{7}$ truncation, we conjecture that the Freund-Rubin term is given by the potential for the scalars of the truncated $d=4$ supergravity and a variation of the potential. While the corresponding on-shell conjecture has already been in the literature [21], we propose a formula, (6.8), that bears this conjecture out more concretely (off-shell). A corollary of our conjecture is that for sectors that are purely characterised by pseudoscalar expectation values, the Freund-Rubin term is $y$-independent and is completely given by the scalar potential. We prove the conjecture up to quadratic order in the scalar expectation value and verify it for the $\mathrm{G}_{2}$ invariant sector. In the future, we hope to provide a proof of this conjecture.

The GVPs and fermion supersymmetry transformations provide a new vista on the form of the $D=11$ field strength that arises from uplifting $d=4$ solutions. Given the striking simplicity of the conjectured Freund-Rubin term, a natural question that we can now investigate, arises: do the other components of the field strength take a similarly simple form that depend on very general data of the reduced theory, such as the scalar potential or its derivatives. Another aspect that we would like to investigate is whether the conjectured form of the Freund-Rubin term holds in general for all truncations of any theory. A setting in which the analogous question can be addressed using similar methods (analysis of GVPs and fermion supersymmetry variations) is the reduction of type IIB supergravity to five dimensions, where the necessary framework exists [40] - nonlinear ansätze, which arise from an analysis of the supersymmetry transformations of the vectors [40], have been proposed [41] and presented explicitly [42] in this case. In this case, the analysis of the supersymmetry transformations of the vectors has already been used by Pilch and Warner (appendix A of ref. [43]) to derive uplift formulae for the metric and the dilaton.

Our study of reductions of $D=11$ supergravity to four dimensions shows that consistent truncations seem to have simple, generic features that are obscured by the complexity of particular examples. With duality symmetry as a guide $[1,6]$, we are able to tease out these features and it is hoped that in the future we will learn something very general and conceptually deep about all reductions.

\section{Acknowledgments}

We would like to thank Gary Gibbons, Krzysztof Pilch and Chris Pope for useful discussions. H.G. and M.G. would like to thank the AEI, in particular H.N., as well as the Mitchell Institute, TAMU, in particular Chris Pope, for their generous hospitality. H.G. and M.G. are supported by King's College, Cambridge. H.G. acknowledges funding from the European Research Council under the European Community's Seventh Framework Programme (FP7/2007-2013) / ERC grant agreement no. [247252]. 


\section{A Contractions of $\mathrm{G}_{2}$ invariants with Killing forms}

The $\mathrm{G}_{2}$ invariant tensors can be used to define the following tensors on the round $S^{7}[4]$

$$
\begin{aligned}
\xi_{m} & =\frac{1}{16} C_{+}^{I J K L} K_{m n}^{I J} K^{n K L}, \quad \xi_{m n}=-\frac{1}{16} C_{+}^{I J K L} K_{m}^{I J} K_{n}^{K L}, \quad \xi=\stackrel{\circ}{g}^{m n} \xi_{m n}, \\
\stackrel{\circ}{S n n}_{m} & =\frac{1}{16} C_{-}^{I J K L} K_{[m n}^{I J} K_{p]}^{K L} .
\end{aligned}
$$

We write terms like e.g. $D_{-}^{I J K L} K_{m}^{I J} K_{n p}^{K L}$ in terms of the $S^{7}$ tensors in (A.1). These fulfill the identities

$$
\begin{aligned}
& \xi_{m} \xi_{n}=\left(9-\xi^{2}\right) \stackrel{\circ}{m n}_{m}-6(3-\xi) \xi_{m n}, \quad \xi_{m} \xi^{m}=(21+\xi)(3-\xi), \\
& S^{m n r} S_{p q r}=2 \delta_{p q}^{m n}+\frac{1}{6} \eta^{m n n}{ }_{p q r s t} S^{r s t}, \quad S^{[m n p} S^{q] r s}=\frac{1}{4} \eta^{m n p q[r}{ }_{t u} S^{s] t u}, \quad S^{m[n p} S^{q r] s}=\frac{1}{6} \eta^{n p q r(m}{ }_{t u} S^{s) t u} .
\end{aligned}
$$

Together with the inverse relations of eq. (A.1),

$$
C_{+}^{I J K L}=\frac{1}{6} \xi K_{m}^{[I J} K^{m K L]}-\frac{3}{2} \xi^{m n} K_{m}^{[I J} K_{n}^{K L]}+\frac{1}{12} \xi^{m} K_{m n}^{[I J} K^{n K L]}, \quad S_{m n p}=\frac{1}{16} C_{-}^{I J K L} K_{[m n}^{[I J} K_{p]}^{K L]},
$$

we obtain

$$
\begin{aligned}
& \delta_{K L}^{I J} K^{m I J} K_{n}^{K L}=8 \delta_{n}^{m}, \quad \delta_{K L}^{I J} K_{m n}^{I J} K^{p K L}=0, \quad \delta_{K L}^{I J} K^{m n I J} K_{p q}^{K L}=16 \delta_{p q}^{m n}, \\
& C_{+}^{I J K L} K_{m}^{I J} K_{n}^{K L}=-16 \xi_{m n}, \quad C_{+}^{I J K L} K_{m n}^{I J} K^{p K L}=\frac{16}{3} \xi_{[m} \delta_{n]}^{p}, \\
& C_{+}^{I J K L} K^{m n I J} K_{p q}^{K L}=\frac{32}{3} \xi \delta_{p q}^{m n}-64 \xi_{[p}^{[m} \delta_{q]}^{n]}, \\
& C_{-}^{I J K L} K_{m}^{I J} K_{n}^{K L}=0, \quad C_{-}^{I J K L} K_{m n}^{I J} K_{p}^{K L}=16 S_{m n p}, \quad C_{-}^{I J K L} K_{m n}^{I J} K_{p q}^{K L}=-\frac{8}{3} \stackrel{\vartheta}{m n n p q r s t}^{r s t}, \\
& D_{+}^{I J K L} K_{m}^{I J} K_{n}^{K L}=0, \quad D_{+}^{I J K L} K_{m n}^{I J} K_{p}^{K L}=-48 \xi_{[m}^{q} S_{n p] q}+\frac{16}{3} \xi S_{m n p}+\frac{4}{9} \grave{\eta}_{m n p q r s t} \xi^{q} S^{r s t},
\end{aligned}
$$

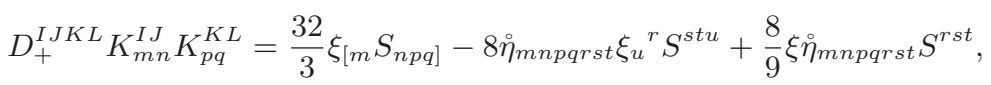

$$
\begin{aligned}
& D_{-}^{I J K L} K_{m}^{I J} K_{n}^{K L}=\frac{16}{3} S_{m n p} \xi^{p}, \\
& D_{-}^{I J K L} K_{m n}^{I J} K_{p}^{K L}=-32 \xi^{q}{ }_{[m} S_{n] p q}+16 S_{m n q} \xi_{p}^{q}+\frac{16}{3} \xi S_{m n p}-\frac{4}{9} \stackrel{\ominus}{\eta n n p q r s t}^{q} \xi^{q} S^{r s t}, \\
& D_{-}^{I J K L} K_{m n}^{I J} K_{p q}^{K L}=\frac{16}{3} \xi_{[m} S_{n] p q}+\frac{16}{3} S_{m n[p} \xi_{q]}-\frac{16}{3} \grave{\vartheta}_{m n[p \mid r s t u} \xi_{q]}^{r} S^{s t u}+\frac{16}{3} \stackrel{\diamond}{[m \mid p q r s t u}_{n n]}^{r} S^{s t u} .
\end{aligned}
$$

\section{B Freund-Rubin term in the $\mathrm{SO}(3) \times \mathrm{SO}(3)$ invariant sector}

The $\mathrm{SO}(3) \times \mathrm{SO}(3)$ invariant sector is given by

$$
\phi_{I J K L}=\frac{\lambda}{2}\left[\cos \alpha\left(Y_{+}^{I J K L}+i Y_{-}^{I J K L}\right)-\sin \alpha\left(Z_{+}^{I J K L}-i Z_{-}^{I J K L}\right)\right],
$$

where $Y_{ \pm}$and $Z_{ \pm}$are $\mathrm{SO}(3) \times \mathrm{SO}(3)$ invariant tensors. 
The scalar potential reads

$$
V(\lambda)=\frac{g^{2}}{2}\left(\tilde{s}^{4}-8 \tilde{s}^{2}-12\right) .
$$

Here, $\tilde{s}=\sinh \sqrt{2} \lambda$ and $\tilde{c}=\cosh \sqrt{2} \lambda$. Note that $V$ does not depend on $\alpha$ [44].

In ref. [30], the $u$ and $v$ tensors are given in terms of $\mathrm{SO}(3) \times \mathrm{SO}(3)$ invariants

$$
\begin{gathered}
Y_{+}^{I J K L}, Z_{+}^{I J K L}: \text { selfdual, } \quad Y_{-}^{I J K L}, Z_{-}^{I J K L}: \text { anti - selfdual, } \\
\Pi^{I J K L}=\frac{1}{8}\left(Y_{+}^{I J M N}+i Y_{-}^{I J M N}\right)\left(Y_{+}^{M N K L}-i Y_{-}^{M N K L}\right)
\end{gathered}
$$

from which we define the following $y$-dependent scalars

$$
\xi(y)=-\frac{1}{16} Y_{I J K L}^{+} K_{m}^{I J} K^{m K L}, \quad \zeta(y)=-\frac{1}{16} Z_{I J K L}^{+} K_{m}^{I J} K^{m K L} .
$$

Using the results in ref. [30] for the $u$ and $v$ tensors and identities stated in that paper, we find

$$
\begin{aligned}
\mathfrak{f}_{F R} & =\frac{m_{7}}{\sqrt{2}}\left(6+4 \tilde{s}^{2}-\frac{\tilde{s}^{4}}{2}\right)+\frac{m_{7}}{6}(\zeta \sin \alpha-\xi \cos \alpha)\left(4 \tilde{s} \tilde{c}-\tilde{s}^{3} \tilde{c}\right) \\
& =\frac{m_{7}}{\sqrt{2} g^{2}}\left(-V(\lambda)-\frac{1}{12}(\zeta \sin \alpha-\xi \cos \alpha) \frac{\mathrm{d} V}{\mathrm{~d} \lambda}\right) .
\end{aligned}
$$

Again the $y$-dependence is contained in $\xi$ and $\zeta$.

\section{Freund-Rubin term in $\mathrm{SU}(4)^{-}$invariant sector}

The $\mathrm{SU}(4)^{-}$invariant sector is parametrised by a single pseudoscalar expectation value,

$$
\phi_{I J K L}=\frac{1}{2} i \lambda Y_{-}^{I J K L} .
$$

In this case, we find that

$$
\mathfrak{f}_{F R}=-\sqrt{2} m_{7} c^{2}\left(c^{2}-4\right)
$$

for $c=\cosh 2 \lambda$. We note that, since this sector only contains a pseudoscalar, i.e. there are no selfdual tensors, the Freund-Rubin term is indeed $y$-independent even away from the stationary point.

Open Access. This article is distributed under the terms of the Creative Commons Attribution License (CC-BY 4.0), which permits any use, distribution and reproduction in any medium, provided the original author(s) and source are credited.

\section{References}

[1] H. Godazgar, M. Godazgar and H. Nicolai, Generalised geometry from the ground up, JHEP 02 (2014) 075 [arXiv:1307.8295] [INSPIRE]. 
[2] H. Godazgar, M. Godazgar and H. Nicolai, Nonlinear Kaluza-Klein theory for dual fields, Phys. Rev. D 88 (2013) 125002 [arXiv: 1309.0266] [InSPIRE].

[3] B. de Wit and H. Nicolai, The consistency of the $S^{7}$ truncation in $D=11$ supergravity, Nucl. Phys. B 281 (1987) 211 [InSPIRE].

[4] B. de Wit, H. Nicolai and N.P. Warner, The embedding of gauged $N=8$ supergravity into $d=11$ supergravity, Nucl. Phys. B 255 (1985) 29 [INSPIRE].

[5] B. de Wit and H. Nicolai, Deformations of gauged $\mathrm{SO}(8)$ supergravity and supergravity in eleven dimensions, JHEP 05 (2013) 077 [arXiv: 1302.6219] [INSPIRE].

[6] B. de Wit and H. Nicolai, $d=11$ supergravity with local $\mathrm{SU}(8)$ invariance, Nucl. Phys. B 274 (1986) 363 [InSPIRE].

[7] E. Cremmer, B. Julia and J. Scherk, Supergravity theory in eleven-dimensions, Phys. Lett. B 76 (1978) 409 [INSPIRE].

[8] H. Nicolai and H. Samtleben, Maximal gauged supergravity in three-dimensions, Phys. Rev. Lett. 86 (2001) 1686 [hep-th/0010076] [INSPIRE].

[9] H. Nicolai and H. Samtleben, Compact and noncompact gauged maximal supergravities in three-dimensions, JHEP 04 (2001) 022 [hep-th/0103032] [INSPIRE].

[10] B. de Wit, H. Samtleben and M. Trigiante, On Lagrangians and gaugings of maximal supergravities, Nucl. Phys. B 655 (2003) 93 [hep-th/0212239] [INSPIRE].

[11] B. de Wit, H. Samtleben and M. Trigiante, Gauging maximal supergravities, Fortsch. Phys. 52 (2004) 489 [hep-th/0311225] [INSPIRE].

[12] B. de Wit, H. Samtleben and M. Trigiante, The maximal $D=4$ supergravities, JHEP 06 (2007) 049 [arXiv:0705.2101] [INSPIRE].

[13] E. Cremmer and B. Julia, The $N=8$ supergravity theory. 1. The Lagrangian, Phys. Lett. B 80 (1978) 48 [INSPIRE].

[14] E. Cremmer and B. Julia, The SO(8) supergravity, Nucl. Phys. B 159 (1979) 141 [inSPIRE].

[15] H. Godazgar, M. Godazgar and H. Nicolai, Testing the non-linear flux ansatz for maximal supergravity, Phys. Rev. D 87 (2013) 085038 [arXiv:1303.1013] [InSPIRE].

[16] B. de Wit and H. Nicolai, $N=8$ supergravity, Nucl. Phys. B 208 (1982) 323 [INSPIRE].

[17] G. Dall'Agata, G. Inverso and M. Trigiante, Evidence for a family of $\mathrm{SO}(8)$ gauged supergravity theories, Phys. Rev. Lett. 109 (2012) 201301 [arXiv:1209.0760] [INSPIRE].

[18] G. Dall'Agata, G. Inverso and A. Marrani, Symplectic deformations of gauged maximal supergravity, JHEP 07 (2014) 133 [arXiv:1405.2437] [INSPIRE].

[19] H. Godazgar, M. Godazgar and H. Nicolai, Embedding tensor of Scherk-Schwarz flux compactifications from eleven dimensions, Phys. Rev. D 89 (2014) 045009 [arXiv:1312.1061] [INSPIRE].

[20] K. Lee, C. Strickland-Constable and D. Waldram, New gaugings and non-geometry, arXiv: 1506.03457 [INSPIRE].

[21] H. Nicolai and K. Pilch, Consistent truncation of $D=11$ supergravity on $A d S_{4} \times S^{7}$, JHEP 03 (2012) 099 [arXiv:1112.6131] [INSPIRE].

[22] P.G.O. Freund and M.A. Rubin, Dynamics of dimensional reduction, Phys. Lett. B 97 (1980) 233 [INSPIRE].

[23] M. Duff and C. Pope, Kaluza-Klein supergravity and the seven sphere, lectures given at September school on supergravity and supersymmetry, Trieste Italy September 6-18 1982 [INSPIRE]. 
[24] A. Coimbra, C. Strickland-Constable and D. Waldram, $E_{d(d)} \times \mathbb{R}^{+}$generalised geometry, connections and M-theory, JHEP 02 (2014) 054 [arXiv: 1112.3989] [INSPIRE].

[25] B. de Wit and H. Nicolai, The parallelizing $S_{7}$ torsion in gauged $N=8$ supergravity, Nucl. Phys. B 231 (1984) 506 [InSPIRE].

[26] H. Nastase, D. Vaman and P. van Nieuwenhuizen, Consistent nonlinear KK reduction of $11 D$ supergravity on $A d S_{7} \times S^{4}$ and selfduality in odd dimensions, Phys. Lett. B 469 (1999) 96 [hep-th/9905075] [INSPIRE].

[27] H. Nastase, D. Vaman and P. van Nieuwenhuizen, Consistency of the $A d S_{7} \times S^{4}$ reduction and the origin of selfduality in odd dimensions, Nucl. Phys. B 581 (2000) 179 [hep-th/9911238] [INSPIRE].

[28] M. Cvetič, H. Lü and C.N. Pope, Geometry of the embedding of supergravity scalar manifolds in $D=11$ and $D=10$, Nucl. Phys. B 584 (2000) 149 [hep-th/0002099] [INSPIRE].

[29] R. Corrado, K. Pilch and N.P. Warner, An $N=2$ supersymmetric membrane flow, Nucl. Phys. B 629 (2002) 74 [hep-th/0107220] [InSPIRE].

[30] H. Godazgar, M. Godazgar, O. Krüger, H. Nicolai and K. Pilch, An $\mathrm{SO}(3) \times \mathrm{SO}(3)$ invariant solution of $D=11$ supergravity, JHEP 01 (2015) 056 [arXiv:1410.5090] [INSPIRE].

[31] K. Pilch, A. Tyukov and N.P. Warner, Flowing to higher dimensions: a new strongly-coupled phase on M2 branes, arXiv:1506.01045 [INSPIRE].

[32] C. Hillmann, Generalized $E_{7(7)}$ coset dynamics and $D=11$ supergravity, JHEP 03 (2009) 135 [arXiv:0901.1581] [INSPIRE].

[33] G. Bossard, C. Hillmann and H. Nicolai, $E_{7(7)}$ symmetry in perturbatively quantised $N=8$ supergravity, JHEP 12 (2010) 052 [arXiv:1007.5472] [INSPIRE].

[34] H. Godazgar, M. Godazgar, O. Hohm, H. Nicolai and H. Samtleben, Supersymmetric $E_{7(7)}$ exceptional field theory, JHEP 09 (2014) 044 [arXiv:1406.3235] [INSPIRE].

[35] O. Hohm and H. Samtleben, Exceptional field theory. II. $E_{7(7)}$, Phys. Rev. D 89 (2014) 066017 [arXiv:1312.4542] [InSPIRE].

[36] H. Godazgar, M. Godazgar and H. Nicolai, Einstein-Cartan calculus for exceptional geometry, JHEP 06 (2014) 021 [arXiv:1401.5984] [INSPIRE].

[37] N.P. Warner, Some new extrema of the scalar potential of gauged $N=8$ supergravity, Phys. Lett. B 128 (1983) 169 [INSPIRE].

[38] N.P. Warner, Some properties of the scalar potential in gauged supergravity theories, Nucl. Phys. B 231 (1984) 250 [INSPIRE].

[39] D.S. Berman, M. Cederwall, A. Kleinschmidt and D.C. Thompson, The gauge structure of generalised diffeomorphisms, JHEP 01 (2013) 064 [arXiv:1208.5884] [INSPIRE].

[40] F. Ciceri, B. de Wit and O. Varela, IIB supergravity and the $E_{6(6)}$ covariant vector-tensor hierarchy, JHEP 04 (2015) 094 [arXiv: 1412.8297] [INSPIRE].

[41] K. Lee, C. Strickland-Constable and D. Waldram, Spheres, generalised parallelisability and consistent truncations, arXiv:1401.3360 [INSPIRE].

[42] A. Baguet, O. Hohm and H. Samtleben, Consistent type IIB reductions to maximal 5D supergravity, Phys. Rev. D 92 (2015) 065004 [arXiv:1506.01385] [InSPIRE].

[43] K. Pilch and N.P. Warner, $N=2$ supersymmetric $R G$ flows and the IIB dilaton, Nucl. Phys. B 594 (2001) 209 [hep-th/0004063] [INSPIRE].

[44] N. Bobev, A. Kundu, K. Pilch and N.P. Warner, Minimal holographic superconductors from maximal supergravity, JHEP 03 (2012) 064 [arXiv:1110.3454] [INSPIRE]. 